\title{
1 Deliberation and enaction during adaptive economic
} 2 choice

David J. Hawellek ${ }^{1,2,3}$, Kevin A. Brown ${ }^{1}$, Bijan Pesaran ${ }^{1 *}$

${ }^{1}$ Center for Neural Science

New York University

New York, NY 10003, USA

$12{ }^{2}$ Center for Integrative Neuroscience

13 University Tübingen

14 Tübingen, 72076, Germany

${ }^{3}$ MEG Center

18 University Tübingen

\section{${ }^{*}$ Correspondence} Bijan Pesaran, Ph.D. 4 Washington PI. Rm 809 New York, NY 10003

26 Tel: 212.998.3578, Fax 212.995.4011

27 E-mail: bijan@nyu.edu

28

29 


\section{Summary}

32 Economic decisions can adapt to contexts. Choices can be quick and impulsive or slow and more

33 deliberative, depending on the temporal context. Choices can also depend on how we enact the

34 choice, in an action context. Where we decide to go for dinner may change if we can take a taxi

35 or need to walk.

37 We hypothesized that frontal action circuits could contribute to adapting economic choices to 38 context because of their privileged position over actions as endpoints of decisions.

40 To test this, we performed an unbiased survey of neuronal population activity across motor, 41 premotor and prefrontal cortices as animals expressed context-dependent economic preferences.

42 Activity in distributed action circuits tracked the animals' evolving preferences in real-time and 43 integrated them with a signal for their enaction. We propose that frontal action circuits form a 44 neural substrate that supports an adaptive control over economic choice by flexibly translating 45 real-time preferences into actions. 


\section{Introduction}

When we make economic decisions, we express subjective preferences. Forming preferences requires a deliberation process that compares different options. Expressing a preference requires an enaction process that translates the deliberation into choices. Together, these two processes - deliberation and enaction - control our decisions. A central feature of our goal-directed behavior

52 is that the control over our decisions is flexible and allows decision making to adapt to contexts ${ }^{1,2}$.

Different temporal contexts often profoundly affect our decisions. Faster decisions tend to be objectively less optimal than slower decisons. This is often observed as a progression from impulsive to more deliberative and even optimal choices as time goes by ${ }^{3-7}$. How we enact decisions also provides an important action context. Our preferences can change depending on how we express them. For example, decisions made with a reach require effort and can show strong spatial biases $^{8}$, while saccades are comparatively effortless and reveal different decision biases $^{9-11}$. In this way, how we enact a choice can itself become part of the decision process. A key question is how neural circuits allow decisions to adapt to changing temporal and action contexts.

Action circuits extend across the frontal and prefrontal cortices and are well situated to track action preferences and translate them into choices in real-time ${ }^{12-14}$. We hypothesized that action circuits could in this way contribute to adapting choices to contexts. Specifically, if a neural circuit supports context-dependent choices, then activity that reflects a preference in one context should reorganize to reflect a different preference as the context changes - a signature of deliberation. Furthermore, to enable flexibility over time, the neural activity should reflect the resolution about whether it is the right time to translate a preference into a choice - a signature of enaction.

72 We trained two monkeys to perform a dynamic decision task in which we manipulated the 73 temporal and action contexts of decisions. Temporal context was manipulated by either instructing 74 animals to make their choice immediately or to wait and make their choice after a delay. Action 75 context was manipulated by either instructing animals to make their choice with a reach or a 76 saccade. Behaviorally, each animals choice depended strongly on context. Reach choices were 77 qualitatively different when made immediately or after a delay. Biased and suboptimal immediate 78 reach choices became unbiased and more optimal delayed choices, as if deliberation led to more 79 optimal decisions. In contrast, saccade choices depended less on the temporal context and were 80 more optimal overall. Thus, depending on the action, the preferences of the animals evolved 
81 dynamically, akin to an unfinished and continuous deliberation that could be quickly and flexibly

82 translated into choices at different points in time.

83

84 We performed a large-scale survey of neuronal ensemble activity by systematically making 85 distributed recordings across the dorsal surface of frontal and prefrontal cortices. In line with our 86 hypothesis, distributed frontal ensembles exhibited signals related to the deliberation and 87 enaction that supported the context-dependent choices of the animals. Action related circuits, 88 prominently the dorsal premotor cortex, tracked the preferences of the animals in real-time and 89 combined them with an enaction signal that could flexibly propagate preferences into choices. In 90 sum, we contribute key evidence to support the idea that frontal action circuits can exert control 91 over economic choice that allows adapting choices to contexts. 


\section{Dynamic decisions with common real-world constraints}

96 We trained two monkeys to perform a dynamic foraging task with three critical components (Fig.

97 1a). First, we unpredictably forced the animals to make a decision immediately rather than after

98 a short delay on a third of the trials. Thus, while there was usually a brief moment to come up with

99 a choice, the animals sometimes had to immediately commit to an option. In this way, we

100 introduced two different temporal contexts for making decisions. Second, the reward magnitudes

101 at the movement targets continuously drifted in an uncorrelated way. Thus, the decision behavior

102 relied on continuous learning and could not be based on simple deterministic strategies. Third,

103 the animals sampled the identical environment with either saccades or reaches. The oculomotor

104 system operates under different constraints than the skeletomotor systems. Specifically,

105 saccades are fast and energetically less costly. In this way, we studied how decisions could

106 depend on two different action contexts.

Our goal was to study how neural computations allow economic choice to adapt to such varying contexts, achieving a flexible control of the decisions.

\section{Animals dynamically compute context-dependent preferences}

112 A computational model of choice behavior revealed that animals made qualitatively different reach

113 decisions according to the temporal context. We first used a reinforcement learning framework to 114 infer values from rewards offered during the two-arm bandit decision task ${ }^{15}$. The model obtained 115 predictions about the optimal trial-by-trial value states of the movement targets by using a Kalman 116 filter to predict the sequence of choices from experienced rewards (Fig. 1b). The value predictions 117 made by the computational model correspond to those of an ideal observer and are optimal in 118 that they represent an upper bound on what the animals could have learned through sampling. 119 We refer to these predictions as the objectively optimal values.

121 With this definition of optimality in hand, we observed that animals made qualitatively different 122 decisions depending on both the action context and the temporal context. We found that delayed 123 reaches (DR) were more optimal than immediate reaches (IR). IR were less successful than DR 124 (Hit rate. Monkey J: IR 63.5\%, DR 67.1\%, $p=.02$; Monkey H: IR 69.4\%, DR 73.6\% high value 125 choices, $p=0.004$, Wilcoxon rank-sum tests). The more optimal behavior of the DR was 
associated with a change in the reach bias of the animals across temporal contexts (Fig. 2a, S1). While IR were biased and suboptimal, choices became gradually more evenly distributed across the two movement options, achieving an objectively more optimal income for DR. Since the temporal context was unpredictable trial-by-trial, this suggests animals revised their choices in real-time, consistent with a form of ongoing deliberation.

In comparison, saccade decisions were objectively more optimal than reach decisions (Overall percentage of hit trials, Monkey J: Reaches 65.3\%, Saccades 82.5\%, $\mathrm{p}<10^{-8}$; Monkey $\mathrm{H}$ : Reaches $71.3 \%$, Saccades $77.3 \%, p=1.4 \times 10^{-8}$, Wilcoxon rank-sum tests). Saccade decisions also exhibited a more stable pattern of choices in the two different temporal contexts of immediate and delayed saccades (IS, DS, Fig. 2b, Fig. S2).

More detailed logistic regression models of the choice behavior based on the Kalman-inferred values and behavioral timing parameters such as reaction times corroborated the evidence for context-dependent decision making. (Fig. S1, S2, Supplementary Results).

Overall the choices of the animals were strongly influenced by the temporal and action contexts of the decisions. Most strikingly, reach choices and not saccade choices were associated with a dynamic change in choice preferences of the animals over time, with qualitatively different decisions made immediately or after a brief delay. These behavioral observations are consistent with the idea that the context-dependent choices are revised online. This indicates that making choices involved a process of ongoing deliberation that was dynamically read out through enaction.

We next measured neural dynamics in frontal and prefrontal cortices to test how neural activity that predicts choice in one context reorganizes as the context changes.

\section{Multiplexed coding of choice and enaction by distributed cortical ensembles}

We simultaneously recorded spiking activity across a $2 \mathrm{~cm}$ extent spanning the frontal and prefrontal cortices using a semi-chronic multielectrode array (SC96, Gray Matter Research, USA, Monkey J: 354 single units, 753 multi units; Monkey H: 96 single units, 664 multiunits). The array covered pre- and post-arcuate cortex including prefrontal cortex (PFC), the dorsal and ventral aspects of premotor cortex (PMd, PMv) as well as parts of somato-motor cortex (SM, Fig. 3a, N units: Monkey J, SM=143, PMd=568, PMv=100, PFC=296; Monkey H, SM=81, PMd=227, 
$P M v=132$, PFC=320). Consistent with known anatomical connections and functional specializations, neural activity across the frontal cortex (SM, PMd, PMv) responded strongly during the immediate and delayed reach tasks (Fig S3) while activity across the PFC most strongly responded during the immediate and delayed saccade tasks (Fig S4).

We used multivariate classification (Linear discriminant analysis - LDA) based on spiking activity to track information about upcoming choices as well as the pending enaction of a choice. We decoded trials based on the animals' upcoming movement choice (choice information, Fig. 3b) or the temporal context, i.e. whether a choice had to be enacted immediately (enaction information, Fig. 3c). Both types of decision related information were widely distributed and could be decoded from nearly all anatomical subdivisions before overt behavior (Fig. 3b, c, Fig S3, S4). We generally observed that the classification performance for saccades compared to reaches was lower (Fig. 3b,c), except for PFC which showed more pronounced activity and more information for saccades (Fig. S4).

A prominent feature during both reach and saccade choices was a brisk global increase in spiking activity after the onset of the choice targets (Fig. S3, S4). This increase in activity was evident across all cortical areas independent of spatial choice or temporal context. The choice information exhibited similar early increases, even for delayed choices for which the enaction of a choice was withheld for the delay. This pattern was particularly pronounced for PMd and was present for both action contexts (Fig. 3b). While choice information increased, neural activity also started to diverge depending on whether or not a decision had to be enacted immediately (Fig. 3c). Neural activity in PMd again robustly contained such enaction information, leading a cascade across areas (Fig. 3c, Fig. S3, S4). Notably, PFC contained only little enaction information, again, despite the slightly varying coverage between the two animals (Fig. 3a) and the reach or saccade context of the decisions (Fig. 3c).

In sum, we observed distributed, dynamic and action context dependent representation of decision related information. Neuronal ensembles tracked information about upcoming choices along with an enaction signal supporting the flexible allocation of choices. choices. What turns suboptimal IR into more optimal DR? 
194 In the dynamic foraging task, rewards for each option change on each trial. The animal's choices

195 may have depended on how long it takes to compute the objectively optimal value for each option.

196 Information about the objectively optimal values may have been weak at the beginning of a trial,

197 built up slowly over time and have been strongest around the time of delayed choices, explaining

198 the revision of choices across temporal contexts. We next investigated the influence of the

199 objectively optimal values on the ensemble dynamics.

\section{Objectively optimal values influence ensemble dynamics early}

202 We pooled simultaneously recorded units into array-wide ensembles to capture the distributed

203 nature of decision related information (Fig S5a, b, Supplementary Results, Monkey J: 29

204 ensembles, median size 22 units, Monkey H: 27 ensembles, median size 23 units).

We predicted that a larger difference in the Kalman-inferred values between the two targets would lead to better choice classification performance. Specifically, if the objective values were driving the revision of the animals' choices over time, the correlation between single trial value differences and classifier predictions should increase over the course of delayed choice trials. To test this prediction, we regressed the single-trial value differences against single-trial classifier probabilities obtained in 500ms windows centered at four intervals of interest during delayed

212 choice trials (Fig 4; Baseline, Commit, Pre-Go, Reaction Time). We defined the commit time point 213 as the local peak in classification performance within the first 500 ms during delayed choices, i.e.

214 when choice information started to separate depending on whether or not animals enacted a 215 choice. To avoid the confounding influence of choice signals, we performed the regressions 216 separately for ipsilateral and contralateral choices and then averaged the results (see Methods).

218 The Kalman-inferred values significantly influenced the classification of choices throughout the 219 delayed trials for both reaches and saccades (Fig 4, t-tests, $p<0.05$, FDR corrected). Strikingly, 220 the regressions were significant already during the baseline intervals and remained at comparable 221 levels up to the movement period. This observation is in line with the idea that animals predicted 222 the value states of targets in real-time and encoded the optimal value states of the targets at the 223 start of the trial. Further in line with this observation is the finding that saccade choices exhibited 224 a more stable influence of the objective values throughout the trial (Fig 2, Fig S2). Thus, the 225 process of learning and updating objectively optimal values was fast enough to guide the even 226 faster saccade behavior early on and in a stable way. These results do not support the view that 
227 immediate reach choices were suboptimal because information about the Kalman-inferred values

228 built up only slowly in the course of a trial.

229

230 Next to changes in objective value signals, choice revisions may result from a dynamic change in

231 other bias signals that together constitute the animals' subjective preference. Such bias signals

232 may act on pools of neurons that represent the choice options simultaneously and compete over

233 the mutually exclusive selection for choice ${ }^{16}$. We next investigated whether subjective preferences

234 were tracked through two circuits that represented the choice options.

\section{Action circuits dynamically track subjective preferences}

237 We split the neurons into two groups that encoded the two alternative movement directions and

238 assessed the evolution of their activity during the different choices (Fig 5, see Fig S6 and Fig 239 S7).

In line with a competition over selection for enaction, the two pools of units exhibited dynamic activity profiles of that reflected the animals' choice allocation and tracked their subjective preferences. For reaches the pool of units encoding ipsilateral movements was enhanced early during the trials during the baseline and up to the commit, agreeing with the ipsilateral behavioral bias for immediate reaches (Fig 6). For delayed reach choices, the two pools of neurons gradually evolved toward a more balanced state reflecting the more balanced allocation of choices across the two choice options.

For monkey J's saccade decisions, the activity profiles were more balanced from the beginning of the trials, matching the choice behavior. The brisk increase of activity around the commit was still slightly more pronounced for the ipsilateral pool of neurons, agreeing with a slight ipsilateral choice bias for very fast immediate saccades (see Fig S2). Similarly, early enhanced activity of the pool encoding contralateral movements for monkey H's saccades matched a contralateral

254 choice bias.

Overall, context-dependent preferences of the animals were tracked through the activity of 257 competing circuits that represented the available choice options. In other words, the process of 258 deliberation could be read out from action circuits in real-time. 
260 Fast and suboptimal and slow and more optimal decision making is a well described phenomenon

261 in behavioral economics and psychology $y^{3-5,7,17}$. An idea permeating many models of such decision

262 behavior is that dynamic changes in decision making are supported by a transition between

263 different decision systems. In principle, the choice revisions here may have resulted from a

264 transition between two decision systems over time. For example, between a suboptimal model-

265 free system that is biased and/or depends on heuristics and another computationally more

266 demanding model-based system. A prediction of such dual decision system models is that the

267 representational structure of choice across the neuronal ensembles should change over time. We

268 next investigated whether a change in the representational structure across the neuronal

269 ensembles may have been associated with the choice revisions from immediate to delayed

270 decisions with a cross-classification approach.

\section{Context-dependent choices are not associated with a transition between decision systems}

273 We trained classifiers on the ensemble activity at different times during immediate choice trials

274 and predicted delayed choices based on ensemble activity at different times during delayed

275 choice trials. We hyothesized that a transition between decision systems should lead to a

276 difference in the ability to predict delayed choices based on immediate choices.

278 We found significant cross-classification performance between immediate and delayed choices 279 that was changing over time (Fig S9, $p<0.05$, permutation tests, FDR corrected). Cross280 classification ramped up during the baseline period to peak at the commit time point and reaction 281 time periods. In order to assess whether such changes in cross-classification reflect a change in 282 the underlying choice representations, the dynamic changes in classification performance within 283 the immediate and delayed trials need to be accounted for (signal-to-noise ratio). The pattern of 284 cross-classification expected to result purely from the changes in the individual classifier 285 performances is predicted by the product of the classifier performances within immediate and 286 delayed choice. We computed a corrected cross-classification performance (Fig 6) by subtracting 287 this expected pattern from the raw cross-classification performance of each ensemble. The 288 corrected cross-classification revealed both similarities and dissimilarities in the choice 289 representations of immediate and delayed choices (Fig 6, p < 0.05, FDR corrected, permutation 290 tests). A consistently positive corrected cross-classification indicates a surprisingly strong cross291 classification performance and suggests a similarity of the representations between immediate 292 and delayed choices. A consistently negative performance indicates a surprisingly weak cross293 classification, suggesting a dissimilarity of the underlying representations. 
294 A central pattern visible for reaches as well as saccades was that the commit time point reflected

295 a moment in time at which the representational structure of choice pivoted from an early similar

296 representation, present just after the baseline, to a later dissimilar one towards choice execution

297 (Fig 6a, b). This change in representation is in line with the idea of transition from deliberation to 298 enaction. Furthermore, after a choice has been withheld at the commit time point during delayed 299 trials, the representational structure regained similarity with earlier activity. These observations 300 suggest that the neural activity reverted to earlier states during the delay. Importantly, we did not 301 observe significant dissimilar representation when comparing the time after the commit for DR 302 and before the commit for IR. That is, there was no evidence for a change in the representational 303 structure between IR and DR, providing evidence against a transition between decision systems 304 for the revised choices. Instead, IR and DR choices were represented similarly across the 305 recorded ensembles. We repeated the analyses for predicting immediate choices when training 306 on delayed choices and found highly similar patterns (Fig S9)

An observation of note is that monkey J's saccade choices that were associated with the most stable and most strongly objective value driven decision behavior (Fig S2, Table S1) were associated with the most widespread and robust pattern of cross-classification similarity between immediate and delayed choices (Fig 6b). Such stable and robust cross-classification may thus

312 be a signature of a highly stable deliberation process. Along the same lines, the reach crossclassification of both monkeys as well as the saccade cross-classification of monkey $\mathrm{H}$ was less pronounced. Accordingly, the behavior of all these were best modelled containing inversions of reaction time dependent biases (Fig S1, S2, Table S1), suggesting the presence of more complex dynamics with sensorimotor processes contributing to the decisions.

\section{Discussion}

319 Contexts play a central role when primates make decisions. A key question is how neural circuits 320 allow adapting choices to contexts to enable flexible behavior.

322 We find that action circuits multiplex decision signals that reflect a subjective deliberation over 323 actions in real-time and the selection of a choice through enaction ${ }^{18-20}$. When reaching, animals 324 changed their choice preferences on brief time scales such that suboptimal immediate choices 325 turned into more optimal delayed choices. These observations are consistent with the idea that a 326 continued deliberation may have flexibly been read out through the enaction of choices if 327 necessary. Such fast and flexible gating of current action preferences may constitute a form of 
context-dependent control over economic choice at a stage that connects deliberation with enaction. In other words, we observed signatures of control over economic choice most proximal to the endpoint of decisions - action.

332 Previous research in more static decision environments highlights the role of higher level cortical 333 regions such as e.g. the orbitofrontal cortex in the control over economic choice ${ }^{11,21}$. An 334 observation consistent with the idea that high level valuation circuits may fully determine 335 economic decision processes were the saccade choices of monkey J. Monkey J's saccade 336 choices were highly stable over time and reflected the most faithful enaction of objectively optimal values in our data ${ }^{1}$. Such a stable decision process may be most strongly driven through the valuation circuitry. An important implication of the reach decisions reported here is that action contexts may be instrumental to reveal more complex and action dependent choice mechanisms in which actions are part of the decision problem. The computations that support choice are distributed $^{2,12,22-25}$ and extend across several circuits that could provide complementary control over choices in a context-dependent way. Our results are in line with the idea that the context of decisions may define the exact way in which neural circuits exert their control over decisions ${ }^{2}$.

The large-scale electrode arrays allowed for a centimeter scale coverage of peri-arcuate cortex. Nonetheless, a central limitation to our study still the scope of the neural recordings. The contribution of other prefrontal, parietal or subcortical brain structures such as the basal ganglia to the dynamics described here remain unclear, yet. The basal ganglia may supply temporal signals during dynamic decision making that likely constitute a central component of the process of enacting choices ${ }^{19}$. Thus, the full neural mechanism that mediates the flexible gating of choices as observed here may involve more extensive subcortical and cortical networks outside of the scope of our arrays.

Along the same lines, we found that the suboptimal immediate and more optimal delayed reach choices were not associated with a change in the representational structure across the neural ensembles. Such a change in choice substrate for the qualitatively different decisions would have been in line with theories that assume multiple decision systems to underlie the dynamic control 358 of behavior ${ }^{3-5,7,17,26}$. Our results instead suggest that the frontal ensembles contained a more 359 unitary substrate to support the different choices, similar to findings that show a surprising overlap 360 in striatal substrates for behaviorally dissociable types of decision making ${ }^{27}$. However, our findings 361 do not exclude the possibility that the frontal ensembles here constitute a point of convergence of 
separate decision systems for the immediate and delayed reach decisions that extend across other parts of the brain.

The observation that the objective values influenced the ensemble dynamics similarly throughout the trials suggests that factors that are intrinsic to reaching were dynamically adapted over the course of delayed trials to drive differences in decision making. Although the exact nature of these intrinsic bias signals remains unclear, one parsimonious explanation is that the animals dynamically discounted action costs during delayed reach trials to countermand the ipsilateral decision bias. The activity across the frontal ensembles tracked subjective preferences in a way that aggregated such intrinsic changes together with the extrinsic (objectively optimal value) decision factors. An important question for future investigation is how dynamic decision signals intrinsic to actions arise. Motor cortices may present a suitable substrate for the real-time construction of such dynamic action variables themselves. Alternatively they may form loops with other prefrontal cortical regions such as the orbitofrontal cortex or regions that e.g. exhibit effort related signals such as the anterior cingulate cortex ${ }^{28}$ to dynamically control subjective action variables. Importantly, the idea that the frontal ensembles tracked multifaceted preferences in real-time suggests that these circuits are a formidable substrate to support choices that quickly adapt to different decision contexts.

In sum, temporal and action contexts strongly impacted the way that non-human primates made decisions. Action circuits exhibited signatures of decision control by combining signals related to deliberation and enaction enabling a flexible adaption of choices to different contexts.

\section{Methods}

\section{Experimental preparation}

Two male adult rhesus macaques (Macaca mulatta) participated in the study. Monkey $\mathrm{J}$ weighed $10 \mathrm{~kg}$ and Monkey $\mathrm{H}$ weighed $12 \mathrm{~kg}$. All surgical and animal care procedures were approved by the New York University Animal Care and Use Committee and were performed in accordance with US National Institute of Health guidelines for care and use of laboratory animals. The animals had participated in electrophysiological experiments targeting the parietal cortex previous to the experiments presented here and have been trained consistently with established training 392 protocols for this study. Before behavioral training we implanted head-restraint prosthesis. Each 393 monkey was trained in an unlit sound-attenuated electromagnetically shielded room (ETS394 Lindgren). Following behavioral training we implanted customized recording chambers (Gray 395 Matter Research, USA) over the spur of the arcuate sulcus using image-guided sterotaxic surgical 
396 procedures (BrainSight, Rogue Research, Canada). The chambers and microdrives were 397 customized to accommodate the individual anatomy of the animals. During surgery, we measured 398 the implanted locations of the recording chambers, enabling the co-registration of .5mm isotropic 399 T1 weighted (MPRAGE) magnetic resonance images and anatomical information with the 400 electrode array. A SC96 semi-chronic microdrive array (Gray Matter Research, USA) was inserted 401 into the recording chambers and sealed for the duration of the recordings. Before recordings were 402 made during the task behavior, about 10-20 electrodes per day were lowered out of the drive to 403 a position past the dura mater where neural activity could initially be observed. Experimental 404 recordings started from these initial positions and on any recording day up to 20 electrodes were 405 moved in small $(15-60 \mu \mathrm{m})$ increments in a random and distributed pattern to allow for continuous 406 sampling of spiking activity during the recordings. Electrode movement was stopped when spiking 407 activity was encountered and spiking activity was not screened for particular functional profiles 408 before the start of the recordings on each day, leading to unbiased sampling of spiking activity across the array.

Experimental hardware and data acquisition

412 Eye positions were continuously monitored with an infrared eye tracking system at $120 \mathrm{~Hz}$ 413 (ISCAN, USA) and reach touches were registered using an acoustic touch screen (ELO Touch 414 Systems, USA). Both signals were digitized at $1 \mathrm{kHz}$. Visual stimuli were presented on a LCD 415 monitor (Dell) placed at about 40cm from the subjects' eyes immediately behind the touch screen. 416 The experimental environment was controlled with custom written LabView (National Instruments) 417 software on a real-time embedded system (NI PXI-9194, National Instruments). Behavioral events 418 were synchronized with neural recordings using photodiode signals on the bottom corner of the 419 LCD monitor. Fluid rewards were delivered with an electronically controlled solenoid.

421 The microdrive arrays were equipped with glass coated tungsten electrodes (Alpha-Omega, .7$4221.2 \mathrm{M} \Omega$ impedance at $1 \mathrm{kHz}$ ) of variable length conforming to the individual customization of the 423 microdrives and allowing for an overall travel distance (throw) of $2 \mathrm{~cm}$ per electrode. The drives 424 contained 96 electrodes spaced at $1.5 \mathrm{~mm}$ and allowed for individual, bidirectional manual control 425 of electrode movement along one cardinal axis with an approximate maximal resolution of $15 \mu \mathrm{m}$. 426 During the neural recordings signals were amplified, low-pass filtered at $6 \mathrm{kHz}$ and digitized at 30 $427 \mathrm{kHz}$ using 16 bit resolution with the lowest significant bit equal to $0.1 \mu \mathrm{V}$ (NSpike NDAQ System, 428 Harvard Instrumentation Lab, USA; x10 gain headstage, Multichannel Systems, Germany) and 429 streamed to disc (custom $C$ and MATLAB code). Recordings were referenced to separately 
430 implanted ground screws at centimeter distances from the drives that were in contact with the

431 dura mater.

432

433 From the raw signals we obtained multi-unit activity offline by high-pass filtering the data at 300

$434 \mathrm{~Hz}$ and thresholding with a median-based robust threshold estimate of 3.5 standard deviations

435 below signal mean. Single unit activity was obtained by performing principal component analysis

436 on the multi-unit spike waveforms, following by over-clustering of the first three dimensions by k-

437 means and cluster merging through visual inspection using custom code written in MATLAB (The

438 Mathworks, USA). Unit clusters were tracked in successive 100s data windows to account for

439 non-stationarity in the recordings. All multi and single unit activity was visually inspected based

440 on activity profiles during the tasks and only entered into the data base for the analyses presented

441 here when neural responses and stability of the recordings was observed.

Behavioral tasks

444 Animals were trained to perform movement tasks involving reaching while maintaining fixation as 445 well as making saccades while maintaining central touch on a touch screen. On every recording 446 day, animals first performed variable amounts of simple delayed center-out movements to single 447 targets arranged on a circle at a radial distance of 10 degree visual angle. This data is not further 448 discussed here. After the center-out movement tasks the animals performed the dynamic foraging 449 task as detailed in the main text (Fig 1a). The choice targets were located at an eccentricity of 10 450 degree visual angle and were always located on a horizontal axis. Animals had to maintain touch 451 and/or fixation within 2 degree visual angle of all targets for correct task performance. Upon 452 arriving at the target of choice, animals had to hold their gaze or touch at the location for at least $453300 \mathrm{~ms}$ before fluid rewards were delivered. Successive trials were interrupted by a randomized 454 inter trial interval of $800-1300 \mathrm{~ms}$. Three isoluminant colors were used to instruct the animals 455 about movements. The central target was yellow signaling the animals to acquire touch as well 456 as fixation. The eccentric movement targets were either green or red signaling the animals that 457 either reach and fixate or saccade and touch movements were required respectively. As a go cue 458 the central target dimmed into a darker gray that for clear visibility was less luminant than the 459 colors used for instructing the movements. The reach and saccade conditions alternated in longer 460 blocks of random length on every day (Monkey J: Reaches, mean 102 trials, SD 86 trials; 461 Saccades, mean 127 trials, SD 78 trials; Monkey H: Reaches, mean 164 trials, SD 96 trials; 462 Saccades, mean 186 trials, SD 73 trials). Block transitions were unsignaled (apart from the 463 change in target color) and the starting condition was chosen randomly on every day. Both 
animals performed at least one block of reaches and one block of saccades per recording session. Overall monkey $\mathrm{J}$ performed the task on 62 neural recording sessions for a total of 157 reach and 148 saccade blocks and a total of 34994 trials. Monkey $H$ performed the task on 45 neural recording sessions for a total of 109 reach and 102 saccade blocks and a total of 36949 trials. Monkey $\mathrm{J}$ reached with the left arm and neural recordings were made in the right hemisphere. Monkey $\mathrm{H}$ reached with the right arm and neural recordings were made in the left hemisphere.

\section{Reward Drifts and objectively optimal values}

472 During the dynamic choice task, the reward magnitudes at the two movement target locations 473 drifted in an uncorrelated way according to gaussian random walks. The design of the reward 474 drifts and the Kalman filter predictions were based on work on human reinforcement learning 475 systems ${ }^{15}$. Briefly, we measured the opening times of the solenoid valve for which we could 476 reliably and linearly deliver fluid volumes. Based on these measurements on trial $t$ the reward at 477 target $m$ was specified as an opening time of the valve between $220 \mathrm{~ms}$ and $400 \mathrm{~ms}$ and was 478 drawn from a Gaussian distribution around a mean $\mu_{\mathrm{t}, \mathrm{m}}$ with standard deviation $\sigma_{0} 15.5 \mathrm{~ms}$. On every trial the mean drifted with:

$\mu_{m, t+1}=\lambda \mu_{m, t}+(1-\lambda) \theta+v$

where the decay parameter $\lambda$ was 0.9836 , the decay center $\theta$ was 310 ms and the zero mean Gaussian diffusion noise $v$ had a standard deviation of $\sigma_{n}=11.16 \mathrm{~ms}$. We generated 24 pairs of reward drifts for the choice task. In order to coarsely balance the statistics of the drifts across reaches and saccades as well as the two target locations, we replayed the drifts after several days. We either replayed the same drifts for days on which the animals started out with saccades and had sampled the drift starting with reaches already (or vice versa), or we switched the association of spatial movement target and drift.

To objectively predict value states of the movement targets we used a Kalman filter to track the

492 reward drifts. If movement target $m$ was chosen the predicted mean of the drift was updated 493 according to:

494

495

$$
\mu_{m, t}^{p o s t}=\mu_{m, t}^{p r e}+k_{t} \delta_{t}
$$


497 Where $\delta_{\mathrm{t}}$ is the prediction error given as the difference between received reward $r_{\mathrm{t}}$ and its 498 prediction $\mu_{m, t}^{\text {pre }}$ and $\mathrm{k}_{\mathrm{t}}$ is the learning rate:

499

$500 \quad k_{t}=\frac{\sigma_{m, t}^{p r e}}{\left(\sigma_{m, t}^{p r e}+\sigma_{0}^{2}\right)}$

501

502 Together with the mean the predicted variance of the chosen option was updated according to:

503

$\sigma_{m, t}^{2 p o s t}=\left(1-k_{t}\right) \sigma_{m, t}^{2 p r e}$

505

506 The mean and variance of the unchosen movement target were unaffected and predictions for

507 the next trial for all $m$ were obtained as:

508

509

$\mu_{m, t+1}^{\text {pre }}=\lambda \mu_{m, t}^{\text {post }}+(1-\lambda) \theta$ and $\sigma_{m, t+1}^{2 \text { pre }}=\lambda^{2} \sigma_{m, t}^{2 \text { post }}+\sigma_{n}^{2}$

510

511 We used the predicted means from this tracking process as the objectively optimal value state

512 predictions on any given trial.

513

\section{Behavioral modeling}

515 We used nested model testing based on logistic regressions to investigate the choice behavior of

516 the animals in detail. Specifically, we hierarchically fit all parameter of the model:

$518 \quad \ln \left(\frac{p\left(C_{\text {Ipsi }}\right)}{p\left(C_{\text {Contra }}\right)}\right)=V D(\alpha+\gamma T)-\beta T+\lambda$

519 Where $\mathrm{C}$ represents the choices, VD the difference in objective values at the two targets and T

520 the behavioral timing on any given trial. We compared the models using the Akaike information

521 criterion (AIC) and interpreted AIC differences of 10 as strong evidence for a better fit to the data ${ }^{29}$.

522 We chose the best models for behavior (see Fig S1, S2) based on the AIC as well as the

523 observation that all regressors of the models were a significant $(p<0.05$, see Table S1). We

524 combined trials across recordings sessions to obtain the model fits.

525 
527 We used Linear Discriminant Analysis to classify trials according to movement conditions based

528 on the spiking activity of simultaneously recorded units in frontal cortex ${ }^{30}$. We made binary

529 classifications of the trials either based on the direction of the movements that animals made

530 (ipsilateral vs. contralateral) or the temporal condition of the movements (immediate vs. delayed).

531 We refer to these classification performances as choice and enaction information, respectively.

532 Choice information was computed separately for immediate and delayed trials and all trials were

533 pooled to compute the enaction information. The classifiers were trained based on the single trial

534 activity of simultaneously recorded units (range 1 - 40) within sliding 50 ms windows stepping

535 through time in $10 \mathrm{~ms}$ steps. In all multivariate analyses the identical units and ensembles

536 contributed to the classification for reaches and saccades. For all multivariate and univariate

537 analyses within the anatomical subdivisions we included all units recorded in the respective

538 subdivision (Fig 3, S3, S4, 5, S6, S7). For all multivariate analyses pooling units into array-wide

539 ensembles (Fig 4, 6, S8, S9) we only included ensembles with at least 10 units from which at

540 least 1 unit was present in each anatomical subdivision (see Fig S5 a, b). We excluded units from

541 the array wide ensembles if their inclusion would have reduced the number of trials for that

542 ensemble by 100 e.g. due to loss of unit isolation. Classification was only performed if at least 5

543 trials of each movement condition were available and each trial was held out for training when

544 classified, training only on all other trials (leave-one-out cross validation). For the classification

545 results presented in main text figure 3 we only included data of each trial up to 50 ms before its

546 measured reaction time (end of central touch or fixation). We assessed classification performance

547 as the difference in true positive and false positive rates of the classifiers to account for eventual

548 differences in trial numbers between the conditions. To test for significant classification

549 performance, we performed random permutation tests. We obtained resample distributions of

550 classification performances under the null hypothesis by randomly flipping the sign of the

551 classification performances of individual ensembles 10,000 times. We then used these resample

552 distributions to non-parametrically compute $p$-values. Where appropriate we corrected for multiple

553 comparisons by controlling the false discovery rate ${ }^{31}$.

554 We estimated the latencies of the enaction information as the timepoint at which the information

555 reached half maximum within $200-400$ ms after target onset. We only estimated latencies for an

556 anatomical subdivision if significant classification performance was present. We obtained

557 confidence estimates of the latencies by bootstrapping.

558 To regress single trial value differences against the choice classification performance we obtained

559 single trial classification probabilities (posterior class probabilities) according to the procedures

560 described above for average spiking activity within 500 ms windows centered at 4 non- 
561 overlapping time points of interest during delayed choice trials. We defined the baseline time-

562 point as $250 \mathrm{~ms}$ before the target onset, the commit time-point as the local peak in classification

563 performance within the first $500 \mathrm{~ms}$ during delayed choices (i.e. animal and movement specific),

564 the pre-go time point as 250 ms before the go cue on delayed choices and the reaction time time-

565 point as the median reaction time on delayed choices (i.e. animal and movement specific). We

566 regressed the value differences between the two targets and the classification probabilities

567 separately for ipsilateral and contralateral movements using Pearson correlations. To assess the

568 significance of the regressions we performed random permutation tests by shuffling the trial

569 association of value differences and classifier probabilities 10,000 times. We then squared the

570 regression coefficients as well as the resample distribution and non-parametrically converted the

571 data to the corresponding z-scores and averaged the z-scores for each ensemble across

572 ipsilateral and contralateral movements. We then tested whether the average z-scores across

573 ensembles were significantly positive using t-tests.

574 For the cross-classification analyses the classifiers were trained separately on either all

575 immediate choice or all delayed choice trials to predict either delayed or immediate choices,

576 respectively. We obtained corrected cross-classification performance by subtracting the product

577 of the classification performance on delayed and immediate choices for each specific time-point

578 combination of every classification. We assessed significance of the cross-classification

579 performances with permutation tests as described for the within condition classifications above.

580 To assess the dynamics of how action plans are represented across the frontal cortical ensembles

581 we split all neurons into two pools encoding choices towards the ipsilateral or contralateral side,

582 respectively. For this analysis we selected all units that exhibited a significant firing rate difference

583 between ipsilateral and contralateral choices within a 500ms time window before the reaction

584 time. After that we split neurons into pools encoding for the ipsilateral and contralateral movement

585 direction according to the area under the curve of a receiver operating characteristic (AUROC),

586 computed for every unit with ipsilateral movements as the consistent positive class. Units with

587 values greater than 0.5 were treated as the ipsilateral encoding pool and units with values less

588 than 0.5 were treated as the contralateral encoding pool. Before averaging the activity of the units

589 during ipsilateral and contralateral choices we normalized the firing rate of each unit by dividing

590 through the maximum firing rate across all conditions (ipsi- and contralateral movements as well

591 as immediate and delayed trials). Error bars throughout all analyses represent $95 \%$ confidence

592 intervals obtained by bootstrapping. Analyses were performed using MATLAB (The Mathworks,

593 USA). 


\section{References}

1. Padoa-Schioppa, C. \& Conen, K. E. Orbitofrontal Cortex: A Neural Circuit for Economic

Decisions. Neuron 96, 736-754 (2017).

2. Cisek, P. Making decisions through a distributed consensus. Curr. Opin. Neurobiol. 22, 927-936 (2012).

3. O'Donoghue, T. \& Rabin, M. Doing it now or later. Am. Econ. Rev. 89, 103-124 (1999).

4. Thaler, R. H. \& Shefrin, H. An economic theory of self-contol. Journal of Political Economy 89, 392-406 (1981).

5. Loewenstein, G. Out of Control: Visceral Influence on Behavior. Organ. Behav. Hum. Decis. Process. 65, 272-292 (1996).

6. Resulaj, A., Kiani, R., Wolpert, D. M. \& Shadlen, M. N. Changes of mind in decisionmaking. Nature 461, 263-266 (2009).

7. Kahneman, D. Maps of Bounded Rationality: Psychology for Behavioral Economics. Am. Econ. Rev. 93, 1449-1475 (2003).

8. Cos, I., Belanger, N. \& Cisek, P. The influence of predicted arm biomechanics on decision making. J. Neurophysiol. 105, 3022-3033 (2011).

9. Roitman, J. D. \& Shadlen, M. N. Response of neurons in the lateral intraparietal area during a combined visual discrimination reaction time task. J. Neurosci. 22, 9475-89 (2002).

10. Platt, M. L. \& Glimcher, P. W. Neural correlates of decision variables in parietal cortex. Nature 400, 233-238 (1999).

11. Padoa-Schioppa, C. \& Assad, J. A. Neurons in the orbitofrontal cortex encode economic value. Nature 441, 223-226 (2006).

12. Pesaran, B., Nelson, M. J. \& Andersen, R. A. Free choice activates a decision circuit between frontal and parietal cortex. Nature 453, 406-409 (2008).

13. Cisek, P. \& Kalaska, J. F. Neural mechanisms for interacting with a world full of action choices. Annu. Rev. Neurosci. 33, 269-298 (2010).

14. Pesaran, B. Neural correlations, decisions, and actions. Curr. Opin. Neurobiol. 20, 166171 (2010).

15. Daw, N. D., O’Doherty, J. P., Dayan, P., Seymour, B. \& Dolan, R. J. Cortical substrates for exploratory decisions in humans. Nature 441, 876-9 (2006).

16. Cisek, P. Cortical mechanisms of action selection: the affordance competition hypothesis. Philos. Trans. R. Soc. Lond. B. Biol. Sci. 362, 1585-99 (2007).

17. Daw, N. D., Niv, Y. \& Dayan, P. Uncertainty-based competition between prefrontal and dorsolateral striatal systems for behavioral control. Nat. Neurosci. 8, 1704-1711 (2005).

18. Thura, D. \& Cisek, P. Deliberation and commitment in the premotor and primary motor cortex during dynamic decision making. Neuron 81, 1401-1416 (2014).

19. Thura, D. \& Cisek, P. The Basal Ganglia Do Not Select Reach Targets but Control the Urgency of Commitment. Neuron 95, 1160-1170.e5 (2017).

20. Hanes, D. P. \& Schall, J. D. Neural control of voluntary movement initiation. Science 274, 427-30 (1996).

21. Rich, E. L. \& Wallis, J. D. Decoding subjective decisions from orbitofrontal cortex. Nat. Neurosci. 19, 973-980 (2016).

22. Wong, Y. T., Fabiszak, M. M., Novikov, Y., Daw, N. D. \& Pesaran, B. Coherent neuronal ensembles are rapidly recruited when making a look-reach decision. Nat. Neurosci. 19, 327-334 (2016). 
644 23. Hawellek, D. J., Wong, Y. T. \& Pesaran, B. Temporal coding of reward-guided choice in the posterior parietal cortex. Proc. Natl. Acad. Sci. U. S. A. 113, 13492-13497 (2016).

24. Siegel, M., Buschman, T. J. \& Miller, E. K. Cortical information flow during flexible sensorimotor decisions. Science 348, 1352-1355 (2015).

25. Kable, J. W. \& Glimcher, P. W. The neurobiology of decision: consensus and controversy. Neuron 63, 733-45 (2009).

26. Rangel, A., Camerer, C. \& Montague, P. R. A framework for studying the neurobiology of value-based decision making. Nat. Rev. Neurosci. 9, 545-556 (2008).

27. Daw, N. D., Gershman, S. J., Seymour, B., Dayan, P. \& Dolan, R. J. Model-based influences on humans' choices and striatal prediction errors. Neuron 69, 1204-1215 (2011).

28. Walton, M. E., Rudebeck, P. H., Bannerman, D. M. \& Rushworth, M. F. S. Calculating the cost of acting in frontal cortex. Ann. N. Y. Acad. Sci. 1104, 340-356 (2007).

29. Burnham, K. P. \& Anderson, D. R. Model Selection and Multimodel Inference - A Practical Information-Theoretic Approach. (Springer, New York, 1998).

30. Hastie, T., Tibshirani, R. \& Friedman, J. The Elements of Statistical Learning. (Springer, 2016).

31. Benjamini, Y. \& Hochberg, Y. Controlling the False Discovery Rate: A Practical and Powerful Approach to Multiple Testing. J. R. Stat. Soc. Ser. B 57, 289-300 (1995). 
Figure 1: Dynamic foreaging task. a) Animals started a trial by acquiring central touch and fixation.

668 Two diametrically opposed movement targets appeared on a horizontal axis. On 2/3 of all trials 669 the animals had to wait until the dimming of the central stimulus indicated signal to go (Delayed 670 choice). The animals were free to choose either movement target to obtain a fluid reward. On 1/3 671 of all trials the go cue was given immediately with the appearance of the choice targets. On 672 immediate choice trials the animals had to achieve a reaction time that was shorter than the 673 shortest delay on delayed choice trials. Animals made choices either with a reaching movement 674 while maintaining fixation or with a saccade while maintaining touch. In all subsequent analyses 675 the term ipsilateral is used to refer to the side of the reaching arm. The reach and saccade 676 conditions alternated in longer blocks. b) The fluid magnitudes at the two targets drifted across 677 trials in an uncorrelated way. We matched the statistics of the drifts across days and actions by 678 replaying drifts after a few days, exchanging the association of target and drift and 679 counterbalancing the sequence of reach and saccade blocks (see Methods). We used a Kalman 680 filter to predict value states of the targets based on the animals' choice behavior. These values 681 are referred to as objectively optimal values as detailed in the main text.

683 Figure 2: Animals change their reach preferences dynamically. a) Choices and success for reach 684 decisions of monkey J and monkey H. IR (black) and DR (grey) choice probabilities are shown 685 according to the objectively optimal values at the two target locations. Positive value differences 686 indicate a higher value at the target ipsilateral to the reaching arm. Success is depicted with the 687 hit rate that reflects the probability of choosing the objectively higher valued target for the same 688 choices. Dotted lines depict the probability of choosing both sides equally as well as the chance 689 level of detecting the objectively optimal reward. All error bars depict $95 \%$ confidence intervals. 690 b) Same as a but for saccade choices.

692 Figure 3: Frontal ensembles combine information about choices and their enaction. a) Recording 693 locations. The drives were centered on the spur of the arcuate sulcus covering pre- and post694 arcuate sulcus. Recordings were made in the hemisphere contralateral to the reaching arm. 695 Anatomical locations were derived from co-registration of MRI data to the implanted drives. Grey 696 lines depict sulci. PS, principal sulcus, AS, arcuate sulcus; CS, central; sulcus. Electrode locations 697 are shown as colored dots. Only electrodes that contributed spiking activity to the analyses are 698 shown. Electrode colors depict anatomical subdivisions. SM, somato-motor; PMd, dorsal 
699 premotor cortex; PMv, ventral premotor cortex; PFC, prefrontal cortex. T, target onset; G, go cue.

700 b) Classification performance of ipsilateral and contralateral choices based on spiking activity 701 within the anatomical subdivisions (choice information). TPR, true positive rate; FPR, false 702 positive rate. The left column of panels depicts data for monkey $\mathrm{J}$, the right column depicts data 703 for monkey H. Classification performance is shown separately for delayed and immediate choices 704 as well as reaches and saccades. The data for delayed choices is shown for two alignments, to 705 the target onset as well as the go signal. The transition between the two periods is indicated as a 706 vertical dotted gray line. Note that all analyses were based on the spiking activity of the same 707 neurons. c) Classification performance of immediate and delayed trials based on spiking activity 708 within the anatomical subdivisions (enaction information). Only data up to 50 ms before movement 709 onset was used to perform classification of immediate and delayed choices. The time axis is 710 chosen to show a more detailed view onto the time after target onset. We estimated latencies of 711 information onset as the time point at which the information reached half maximum within the 712 shown time window. Latencies were only estimated if significant classification performance was 713 detected. Small panels above the line plots depict the 95\% confidence interval of the latencies for 714 each anatomical subdivision obtained by bootstrapping. The median latency is depicted as a circle 715 on the classification performances for the anatomical subdivisions.

717 Figure 4: Objective values influence ensemble dynamics early. a) Relationship of single trial value 718 differences and classifier probabilities at four time points during delayed reaches (DR). 719 Correlations are shown as z-scores relative to a resample distribution obtained through random 720 permutations (see Methods). Significant influences of the objective values on the classification 721 performance are depicted as solid circles. All error bars depict 95\% confidence intervals. The 722 baseline window was chosen as the 500 ms preceeding target onset. The commit window was 723 chosen as 500 ms centered on the time point at which choice information peaked during delayed 724 trials before then slightly decreasing. At this time point information continued to rise on immediate 725 choices, signaling the pending enaction. The pre-go window was chosen as the 500 ms preceding 726 the go cue. The reaction time (RT) window was chosen as $500 \mathrm{~ms}$ centered on the median 727 reaction time. T, target onset; G, Go cue. Note that all correlations were performed separately for 728 ipsilateral and contralateral choices to avoid choice signals confounding the analysis. b) Same as 729 a for delayed saccade (DS) choices.

731 Figure 5: Action preferences are tracked through competing circuits. a) Activity profiles of two 732 pools of neurons encoding ipsilateral and contralateral choices through increases in their firing 
733 rate. The histograms show the encoding strength of the two pools of units computed as the

734 AUROC minus 0.5 with ipsilateral choices as consistent positive class. Each unit with negative

735 values increases its firing rate for contralateral choices, each unit with positive values increases

736 its firing rate for ipsilateral choices. Units were selected for the analysis for having a significant

737 difference in their firing rate between ipsilateral and contralateral choices in the 500 ms preceding

738 delayed choice reaction times ( $p<0.05$, ranksum test). Units were then split into the two pools

739 based on the sign of their choice encoding. The colored bar depicts how many neurons of each

740 anatomical subdivision contributed to the analysis. The line plots show the activity of each pool of

741 neurons for ipsilateral (left column) and contralateral (right column) choices as well as immediate

742 (top row) and delayed (bottom row) trials. Spiking activity was normalized by dividing with the

743 maximum activity across all conditions for each neuron separately. DR, delayed reaches; IR,

744 immediate reaches; DS, delayed saccades; IS, immediate saccades. T, target onset; G, go cue.

745 All shadings depict 95\% confidence intervals. b) Same as A for monkey $\mathrm{H}$.

747 Figure 6: Similarity of the representation of choice across array wide ensembles. a) We trained

748 choice classifiers on immediate reach trials and predicted delayed reach choices based on activity

749 in delayed reach trials for different combination of time points. The resulting cross-classification

750 was corrected by subtracting the pattern of classifier performance that would be expected based

751 on the product of training and predicting within immediate or delayed trials, respectively. Negative

752 values indicate a consistently weaker and positive values a consistently better than expected

753 cross-classification performance, indicating dissimilar (green) or similar (purple) neuronal

754 representations between immediate and delayed choices. Dotted lines indicate the four time

755 points of interest throughout the trials for orientation. T, target onset; G, go cue. The data is

756 masked to only show significant time points as colored ( $p<0.05$, FDR corrected). 


\section{Figure 1 (Pesaran)}

a

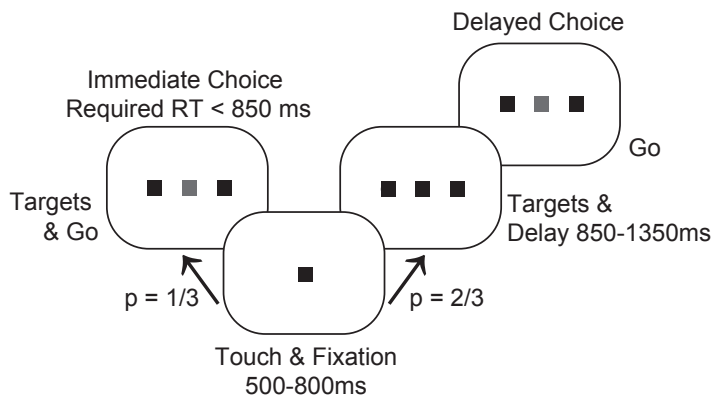

Reward Drifts

Kalman Filter

Value Predictions
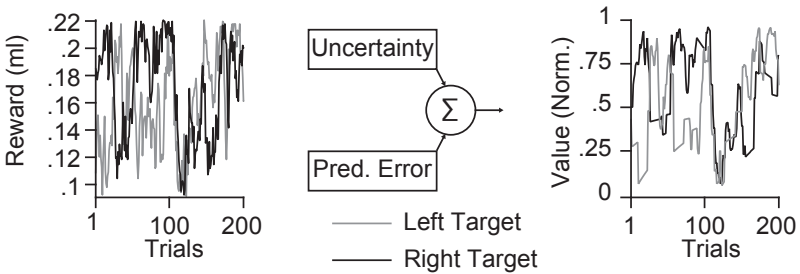


\section{Figure 2 (Pesaran)}

a

Reach choices

Monkey J

Choices

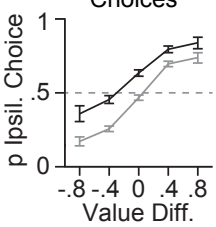

(Ipsil. - Contral.)

Saccade choices

Monkey J

b
Success

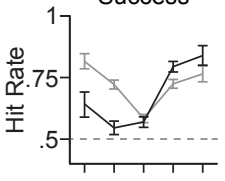

- Immediate
Reach choices Monkey $\mathrm{H}$
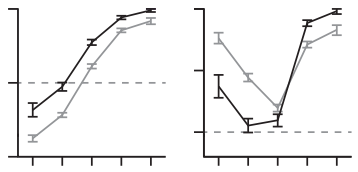

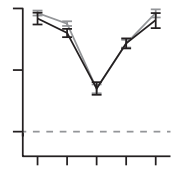

Saccade choices Monkey $\mathrm{H}$
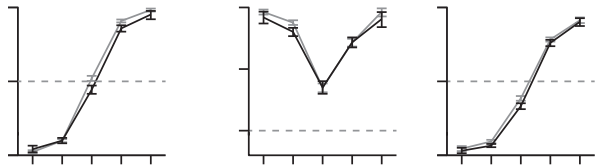

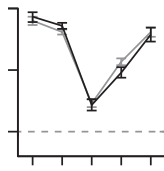


Recording Locations b

Monkey J

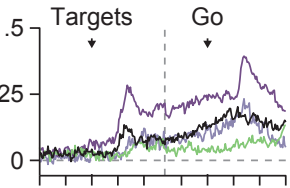

PS

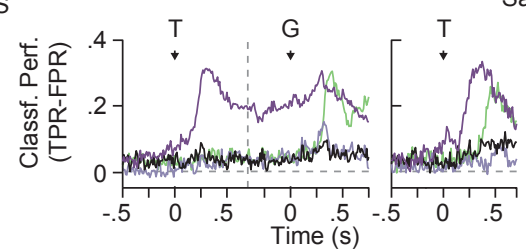

Enaction Information
Choice Information

Reaches

Monkey $\mathrm{H}$

Immediate
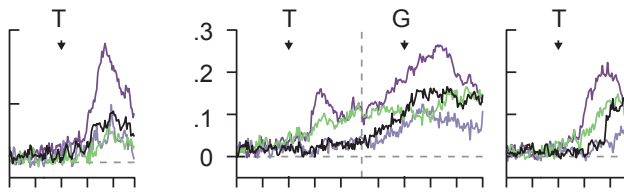

Saccades
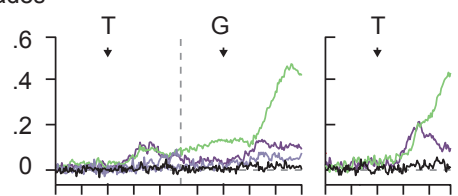

C

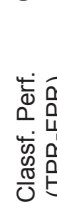

Monkey J

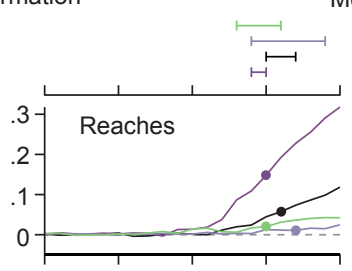

Monkey H
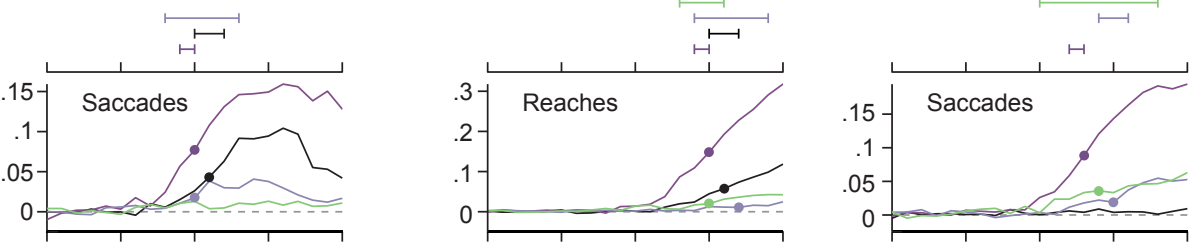


\section{Figure 4 (Pesaran)}

a

Delayed reaches Monkey J

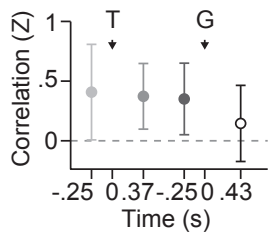

b

Delayed saccades

0
$\mathbb{N}$
0
0
0
0
0
0

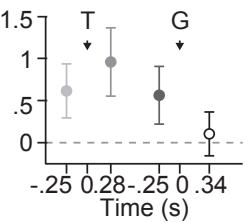

Monkey $\mathrm{H}$

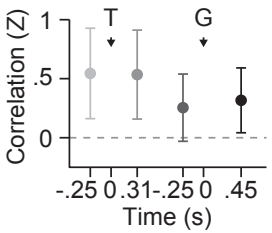

- RT

- $\mathrm{p}_{\mathrm{FDR}}<0.05$

$\bigcirc p_{F D R}>0.05$ 
Figure 5 (Pesaran)

\section{a Reaches} Monkey J Choice Encoding Overall $n=542$

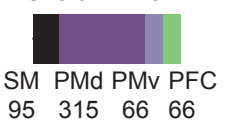
$\begin{array}{llll}95 & 315 \quad 66 \quad 66\end{array}$

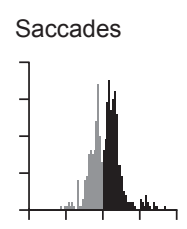

Overall $n=461$

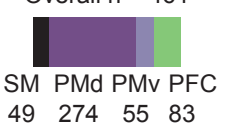

Ipsilateral Choice

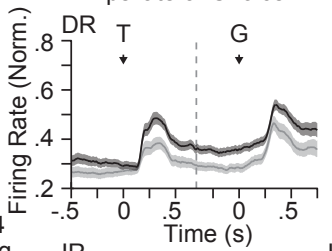

Contralateral Choice
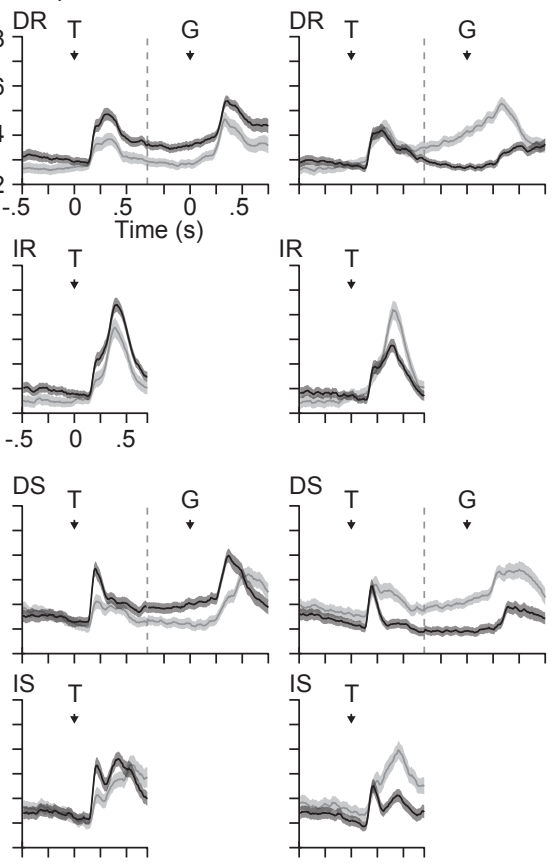

b

Monkey $\mathrm{H}$
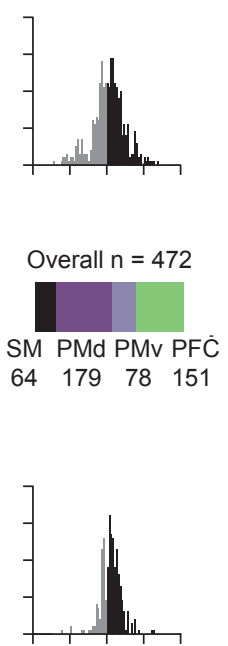

Overall $n=282$

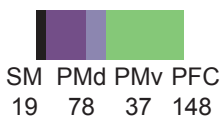

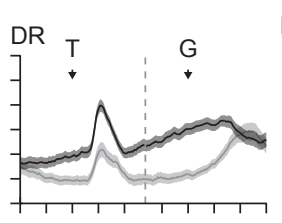

DR
-
-
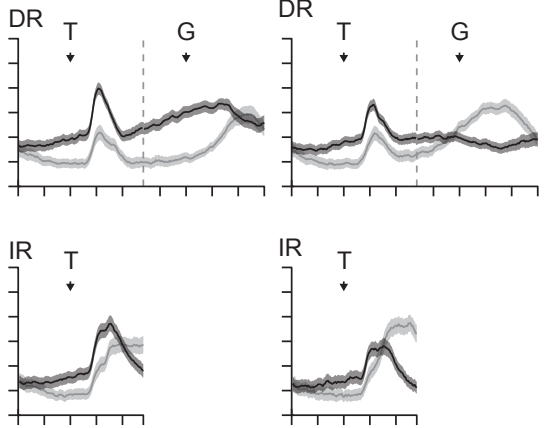

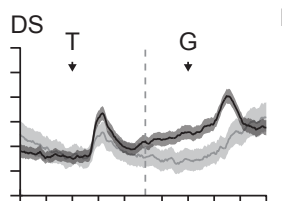

DS

] $\quad$ G
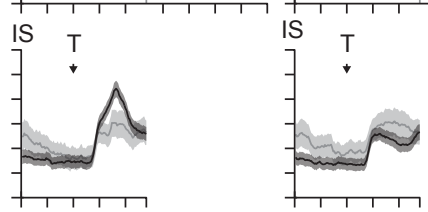


\section{Figure 6 (Pesaran)}

a

Similarity of choice representations

Reaches

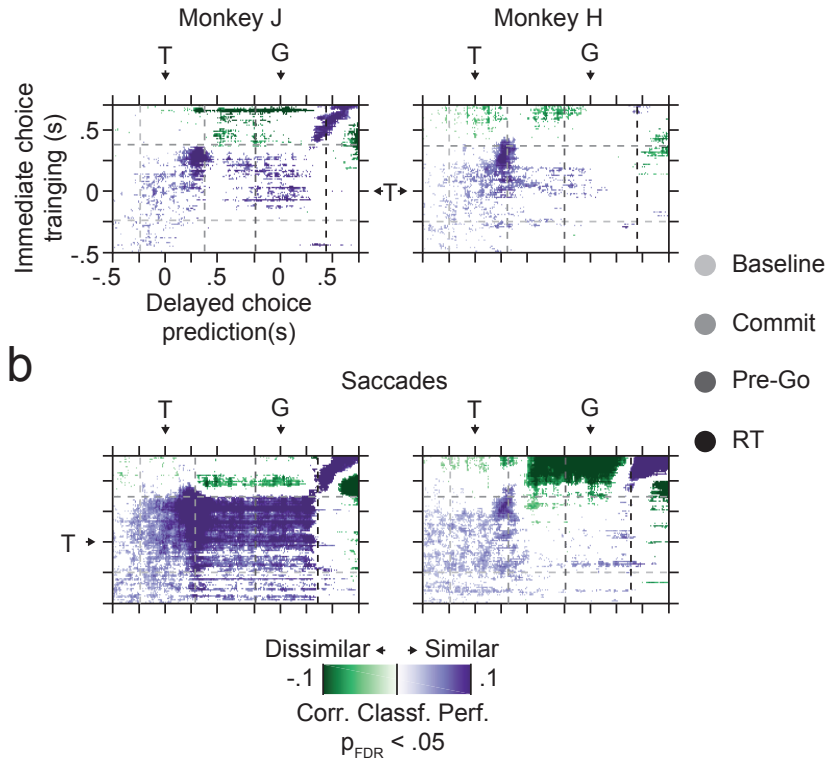




\section{Supplementary Information}

2

3 Deliberation and enaction during adaptive economic 4 choice

5

David J. Hawellek ${ }^{1,2,3}$, Kevin A. Brown ${ }^{1}$, Bijan Pesaran ${ }^{1^{\star}}$

${ }^{1}$ Center for Neural Science

New York University

New York, NY 10003, USA

${ }^{2}$ Center for Integrative Neuroscience

University Tübingen

Tübingen, 72076, Germany

${ }^{3}$ MEG Center

University Tübingen

Tübingen, 72076, Germany 


\section{Supplementary results}

\section{Behavioral modelling of context dependent decision making}

28 To assess how choices were revised in real-time, we modeled the temporal evolution of choice 29 computations from target presentation to the movement choice (Fig S1, S2). The best fitting IR 30 model suffered a significant spatial bias ( $p<0.05$, Table $\mathbf{S 1}$, Fig. $\mathbf{S 1}$ a, b, bottom row). In 31 addition, the way that the animals allocated their IR choices also depended on behavioral timing 32 ( $p<0.05$, Table S1, Fig. S1 a,b) As reaction times got longer the animals allocated their IR 33 choices more evenly across the two movement targets. In other words, fast reaches exhibited the 34 strongest ipsilateral bias while slower reaches sampled the targets more evenly.

The DR exhibited a pattern in which the animals allocated their choices in a more balanced way. As a result, the animals recovered hits on the contralateral side bringing them up to comparable levels with the ipsilateral side (Fig 2 a, Fig. S1 c, d). Strikingly, the timing parameters (reaction time, elapsed time) influenced the allocation of choices in an opposite pattern between IR and DR. That is, the fastest DR reaction times were associated with a contralateral bias that then disappeared for longer reaction times. This effect manifested in opposite signs of the model weights for the reaction time regressors (Table S1).

Overall, animals made qualitatively different reach decisions depending on the temporal context. While IR were suboptimal and biased, choices became gradually more evenly distributed across the two movement options, achieving an objectively more optimal income for DR. Strikingly, the transition from early suboptimal, to later more optimal choices was associated with an inversion of a bias that depended on behavioral timing. Thus, the animals dynamically changed their preferences towards a greater impact of objectively optimal values during DR and this change was linked to dynamics in sensorimotor circuits.

52 The animals engaged in the identical task also with saccades. Saccades are fast and energetically

53 less costly. We predicted that the different dynamics of the oculomotor system may lead to a

54 different pattern of decision making under otherwise identical conditions. Specifically, for 55 saccades the decisions should be less influenced by particular actions themselves and reflect a 56 selection process more closely tied to the objective values of the targets. In line with this 57 prediction, saccade choices of the animals were more stable and optimal than the reach choices 58 (Fig. S2). 
59 For monkey J, IS and DS had a comparable pattern (Fig. 3a) of hits with a symmetrical

60 appearance of hits for both spatial locations (Fig. S2c). The IS choices had a small reaction time

61 dependent bias similar to that of reaches $(p<0.05$.) with a bias towards the ipsilateral side for

62 very fast reaction times that disappeared for longer reaction times (Fig. S2a). The DS of monkey

$63 \mathrm{~J}$ were then strongly driven by the difference in objective values and independent of reaction times

64 (see Table S1, Fig. S2a).

Monkey $\mathrm{H}$ exhibited a comparable foraging pattern with symmetrical hits for IS and DS (Fig. 3b). However, for monkey $\mathrm{H}$ both IS and DS were best modelled with reaction time dependent biases (see Table S1, Fig. S2b). Interestingly, for monkey $\mathrm{H}$ the saccade choices exhibited an inversion of a reaction time dependent bias, similar to what we observed for reaches. A contralateral bias increased with reaction for IS while for DS the contralateral bias decreased with time. The increasing bias for IS choices led to a reaction time dependent decrease in hits on the ipsilateral side (Fig. S2d). That is, with increasing reaction time monkey H's choices were increasingly biased towards the contralateral side. Both monkeys' saccade choices had a slight overall contralateral bias leading to an asymmetry in the hits for the ipsi- and contralateral sides for small value differences (Fig. S2c, d).

Overall the choices of the animals were strongly influenced by the temporal and action contexts of the decisions. Most strikingly, reach choices were associated with a dynamic change in choice preferences of the animals, with qualitatively different decisions made immediately or after a brief delay. These behavioral observations are consistent with the idea that the context dependent choices may have resulted from an ongoing deliberation that was dynamically read out by the process of enaction to support current preferences.

\section{Decision information in array wide neuronal ensembles}

85 We pooled simultaneously recorded units into array wide ensembles to capture the distributed 86 decision information across all recorded areas (Fig S5). Pooling alters the multivariate 87 composition of the ensembles and may lead to different dynamics of information resulting from 88 our classification approach. To reveal the patterns of array wide information, we decoded choice 89 and enaction information (Fig S5c, d). Pooled ensembles captured highly similar dynamical 90 features a compared to the ensembles within individual anatomical subdivisions (Fig 3, S3, S4).

91 Choice information was significantly present during the baseline interval $(p<0.05$, FDR corrected,

92 Fig S5c, d). Choice information exhibited a brisk increase shortly after the onset of the choice 
93 targets and then further increased for immediate choices or slightly dipped and then plateaued

94 for delayed choices. The enaction information also exhibited comparable dynamics and increased

95 sharply at about the time when choice information started to diverge between immediate and

96 delayed choices.

97

98

99 
101 Abbreviations: IR, immediate reach; DR, delayed reach; RT, reaction time; DL, delay; IS, 102 immediate saccade; DS, delayed saccade; AIC, Akaike information criterion.

103 The full model containing all parameters was fit as:

$\ln \left(\frac{p\left(C_{\text {Ipsi }}\right)}{p\left(C_{\text {Contra }}\right)}\right)=V D(\alpha+\gamma T)-\beta T+\lambda$

105 Where $C$ represents choices, VD the difference in objective values at the two options and $T$ the

106 behavioral timing on any given trial. RT is the reaction time, while ET is the elapsed time since

107 target presentation obtained as the delay plus the reaction time.

108

\begin{tabular}{|c|c|c|c|c|}
\hline \multirow[t]{2}{*}{ Monkey J } & \multicolumn{2}{|r|}{ IR, Timing = RT } & \multicolumn{2}{|r|}{ DR, Timing $=\mathrm{ET}$} \\
\hline & AIC & Param. & AIC & Param. \\
\hline constant $(\lambda)$ & 6,680 & 0.5 & 12,947 & -0.1 \\
\hline$\alpha$ & 6,498 & 1.71 & 11,486 & 2.23 \\
\hline$\beta$ & 6,843 & 0.68 & 12,967 & 0.09 \\
\hline constant, $\alpha$ & 6,145 & $0.57,1.85$ & 11,453 & $-0.13,2.25$ \\
\hline constant, $\beta$ & 6,609 & $1.03,1.10$ & 12,934 & $-2.4,-0.3$ \\
\hline constant, $\alpha, \beta$ & 6,084 & $1.08,1.85,1.08$ & 11,441 & $-0.28,2.25,-0.31$ \\
\hline constant, $\alpha, \beta, \gamma$ & 6,069 & $1.14,2.60,1.19,-1.58$ & 11,443 & $-0.28,2.23,-0.31,0.03$ \\
\hline
\end{tabular}

109

\begin{tabular}{|l|c|l|c|l|}
\hline \multirow{2}{*}{ Monkey H } & \multicolumn{2}{|c|}{ IR, Timing = RT } & \multicolumn{2}{c|}{ PR, Timing = ET } \\
\cline { 2 - 5 } & AIC & \multicolumn{1}{|c|}{ Param. } & AIC & Param. \\
\hline \hline constant $(\lambda)$ & 5,805 & 0.97 & 15,311 & 0.26 \\
\hline$\alpha$ & 5,862 & 2.53 & 12,133 & 3.35 \\
\hline$\beta$ & 6,376 & 1.64 & 14,631 & -1.06 \\
\hline constant, $\alpha$ & 4,519 & $1.33,3.43$ & 11,739 & $0.45,3.55$ \\
\hline constant, $\beta$ & 5,691 & $1.52,1.53$ & 13,735 & $-1.38,-3.47$ \\
\hline constant, $\alpha, \beta$ & $\mathbf{4 , 4 5 3}$ & $1.82,3.40,1.36$ & $\mathbf{1 0 , 5 7 1}$ & $-1.22,3.56,-3.51$ \\
\hline constant, $\alpha, \beta, \gamma$ & $\mathbf{4 , 4 5 5}$ & $1.83,3.48,1.40,-0.21$ & 10,573 & $-1.21,3.63,-3.5,-0.15$ \\
\hline Chosen Model, Parameter $\mathrm{p}<0.05$, Parameter $\mathrm{p}>0.05$ \\
\hline \multicolumn{4}{|l}{} \\
\hline
\end{tabular}

\begin{tabular}{|c|c|c|c|c|}
\hline \multirow[t]{2}{*}{ Monkey J } & \multicolumn{2}{|r|}{ IS, Timing = RT } & \multicolumn{2}{|c|}{ DS, Timing = ET } \\
\hline & AIC & Param. & AIC & Param. \\
\hline constant $(\lambda)$ & 8,353 & -0.006 & 15,450 & 0.02 \\
\hline$\alpha$ & 4,941 & 5.50 & 8,461 & 6.41 \\
\hline$\beta$ & 8,272 & 0.46 & 15,488 & -0.06 \\
\hline constant, $\alpha$ & 4,941 & $0.05,5.50$ & 8,454 & $0.82,6.41$ \\
\hline constant, $\beta$ & 7,976 & $0.96,2.16$ & 15,450 & $-0.02,-0.09$ \\
\hline constant, $\alpha, \beta$ & 4,790 & $0.89,5.43,1.87$ & 8,455 & $0.03,6.41,-0.09$ \\
\hline constant, $\alpha, \beta, \gamma$ & 4,786 & $0.90,6.08,1.88,-1.44$ & 8,454 & $0.03,6.06,-0.09,0.73$ \\
\hline
\end{tabular}

\begin{tabular}{|l|c|l|c|l|}
\hline \multirow{2}{*}{ Monkey H } & \multicolumn{2}{|c|}{ IS, Timing = RT } & \multicolumn{2}{c|}{ DS, Timing = ET } \\
\cline { 2 - 5 } & AIC & \multicolumn{1}{|c|}{ Param. } & AIC & Param. \\
\hline \hline constant $(\lambda)$ & 8,457 & -0.4 & 14,898 & -0.23 \\
\hline$\alpha$ & 5,987 & 4.45 & 10,505 & 4.34 \\
\hline$\beta$ & 8,396 & 1.43 & 15,026 & 0.17 \\
\hline constant, $\alpha$ & 5,471 & $-0.76,4.86$ & 10,118 & $-0.49,4.58$ \\
\hline constant, $\beta$ & 8,390 & $-0.13,1.10$ & 14,759 & $-0.63,-0.9$ \\
\hline constant, $\alpha, \beta$ & 5,390 & $-0.30,4.91,1.54$ & 10,088 & $-0.73,4.55,-0.55$ \\
\hline constant, $\alpha, \beta, \gamma$ & $\mathbf{5 , 3 2 1}$ & $-0.49,6.47,0.85,-4.93$ & $\mathbf{1 0 , 0 7 8}$ & $-0.77,5.09,-0.64,-1.18$ \\
\hline \multicolumn{5}{|l|}{ Chosen Model, Parameter $p<0.05$, Parameter $p>0.05$} \\
\hline
\end{tabular}




\section{Supplementary Figure Legends}

112

113 Figure S1: Choice allocation, behavioral modelling and foraging success for reach choices. a) 114 Empirical choices and best model predictions for reach decisions of monkey J. Immediate reach 115 (IR) and delayed reach (DR) choices are shown as a function of behavioral timing and the indicate a higher value at the ipsilateral target, negative value differences indicate a higher value at the contralateral target. IR are shown with reaction time (RT) as timing parameter. DR are shown with elapsed time (ET) since target onset as timing parameter, representing the sum of the delay and RT. The line plots show the choice probabilities based on the two factors separately. All error bars depict $95 \%$ confidence intervals. The best models were chosen according to a nested model selection (see Table S1) and the contributing parameters are shown above the model prediction plots. $\alpha$, value difference; $\beta$, timing; $\gamma$, multiplicative interaction of the value difference and the timing; $\lambda$, constant term. b) Same as a but for monkey $H$. c) Foraging success of monkey $\mathrm{J}$ during reach choices. The hit rate is the probability of the animal choosing the objectively higher valued target. Hit rate is shown for the value and timing factors as well as other conventions described above. d) same as c for monkey $\mathrm{H}$.

Figure S2: Choice allocation, behavioral modelling and foraging success for saccade choices. Same as Fig S1 for saccade choices.

Figure S3: Spiking activity and classification performance within anatomical subdivisions during reach and fixate decisions. a) The left column shows the average spiking activity during the four principal conditions (ipsilateral and contralateral choices as well as immediate and delayed choices) of units within 4 anatomical subdivisions covered by the electrode arrays. The number of contributing units is shown within each panel. T, target onset; G, go cue. The right column shows the classification performance of three classifiers based on the spiking activity. We revealed enaction information as the classification of immediate reaches (IR) vs delayed reaches (DR) and choice information for the IR and DR separately by classifying trials into ipsi- and contralateral movement choices. The classification performances are plotted as thick lines where significant classification performance was detected ( $p<0.05$, FDR corrected, permutation tests). SM, somato-motor; PMd, dorsal premotor cortex; PMv, ventral premotor cortex; PFC, prefrontal cortex. b) Same as A for monkey $\mathrm{H}$. 
145 Figure S4: Spiking activity and classification performance within anatomical subdivisions during

146 saccade and touch decisions. Same as Fig S3 but for saccade and touch choices.

148 Figure S5: Array wide ensemble classification. a) Array wide ensemble composition for monkey

$149 \mathrm{~J}$. The image shows the number of units from each anatomical subdivision. Ensembles are sorted

150 by their size as seen in the marginal plot at the side of the image. The average number of units

151 per ensemble is shown on top of the image plot and a histogram of ensemble sizes is given at

152 the top left. b) same as a for monkey $\mathrm{H}$. c) Classification performances for reach decisions for

153 monkey $\mathrm{J}$ and monkey $\mathrm{H}$. We computed enaction information as the classification performance

154 between immediate and delayed choices and choice information separately for immediate and

155 delayed choices as the classification performance between ipsi- and contralateral movements.

156 Significant classification performance is shown as thick lines $(p<0.05$, FDR corrected,

157 permutation tests). Shadings depict 95\% confidence intervals. TPR, true positive rate; FPR, false

158 positive rate; T, target onset; G, go cue. The cumulative distribution of reaction times (RT) for

159 immediate and delayed choices is shown as dotted lines. d) Same as c but for saccade and touch

160 data. Note that the same ensembles contributed to the classification of reach and saccade data.

162 Figure S6: Sensorimotor circuits track action preferences. Same conventions as Fig 5 but for 163 individual anatomical subdivisions during reach and fixate choices. a) Activity profiles for 164 ipsilateral (left column) and contralateral (right column) reaches for pools of neurons encoding the 165 ipsilateral (black) and contralateral (gray) during delayed (solid lines) and immediate (dotted lines) 166 choices. Each row depicts activity in one anatomical subdivision. See Fig 5 for the number of 167 neurons found to encode choices in each anatomical subdivision. SM, somato-motor; PMd, dorsal 168 premotor cortex; PMv, ventral premotor cortex; PFC, prefrontal cortex. T, target onset; G, go cue; 169 b) Same as A for monkey $\mathrm{H}$.

171 Figure S7: Same as Fig S6 for saccade and touch data.

173 Figure S8: Raw cross-classification a) Classification performance of training classifiers on 174 different time points of immediate choices and predicting delayed choices (left) or vice versa 175 (right). The image plots show significant cross-classification as purple ( $p<0.05$, FDR corrected). 176 The line plots on the sides show slices of the image plot along with $95 \%$ confidence intervals 177 (shadings) along the four time-points of interest during the trials as used previoulsy: baseline, 
178 commit, pre-go and reaction time. The upper row depicts cross-classification of reaching, the

179 bottom row shows cross-classification of saccades. b) Same as a for monkey $\mathrm{H}$.

180

181 Figure S9: Similarity of the choice representations obtained through corrected cross-classification

182 for training on delayed choices and predicting immediate choices. Same conventions as Fig 6. 
Figure S1 (Pesaran)

a

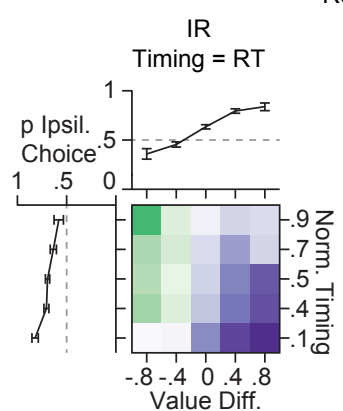

$$
\text { Monkey J }
$$

Model: $\alpha, \beta, \gamma$

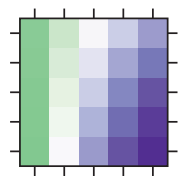

C

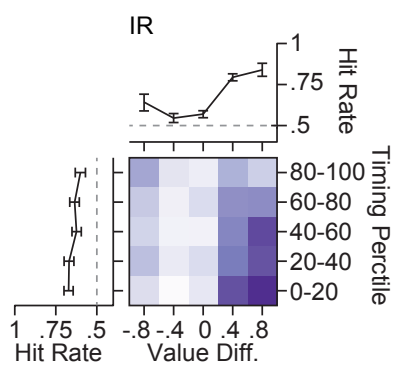

Timing $=\mathrm{ET}$

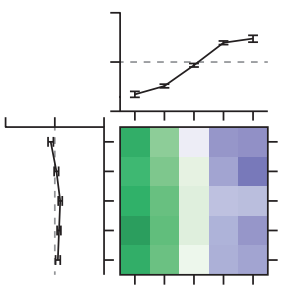

b

IR

Timing $=\mathrm{RT}$

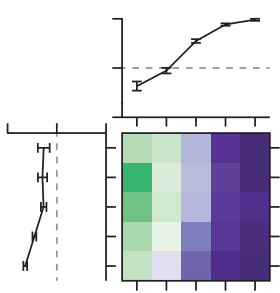

p Ipsil. Choice

Model: $\alpha, \beta, \lambda$
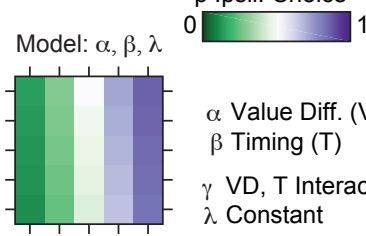

$\alpha$ Value Diff. (VD)

$\beta$ Timing ( $T$ )

$\gamma$ VD, T Interaction

$\lambda$ Constant

Model: $\alpha, \beta, \lambda$
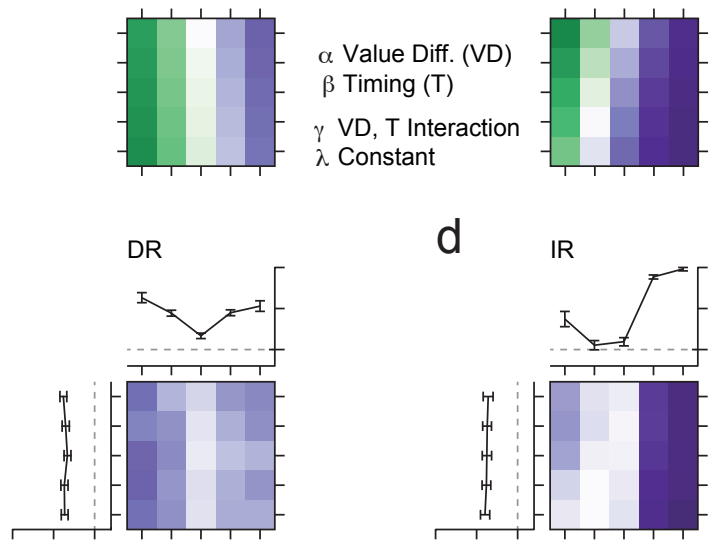

d IR

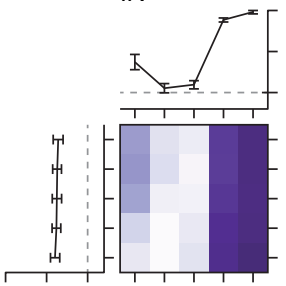

Reach choices Monkey $\mathrm{H}$

DR

Timing $=\mathrm{ET}$

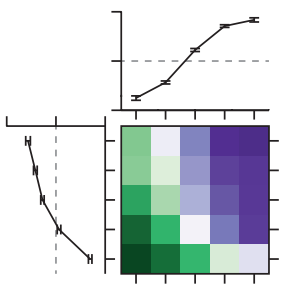

Model: $\alpha, \beta, \lambda$
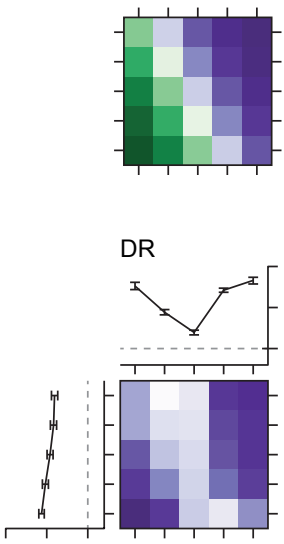
Figure S2 (Pesaran)

a

IS

Saccade choices Monkey J

Timing $=\mathrm{ET}$

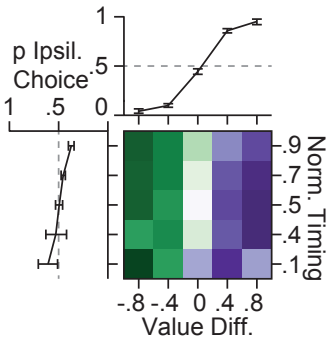

Model: $\alpha, \beta, \lambda$

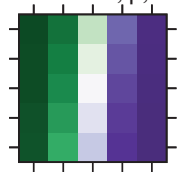

C

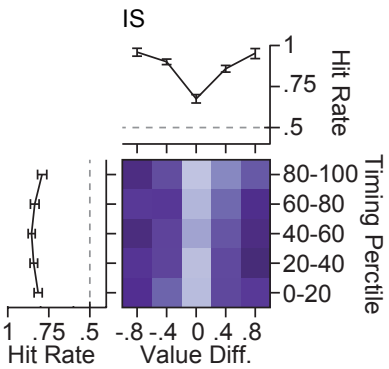

Model: $\alpha, \lambda$ b

\section{Saccade choices}

Monkey $\mathrm{H}$

DS

Timing = ET

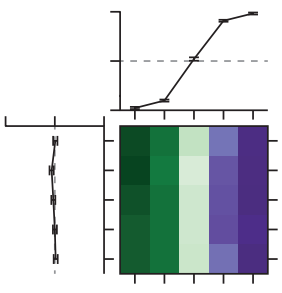

Timing $=\mathrm{R} T$

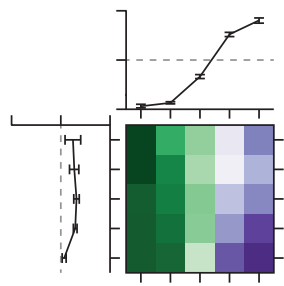

p Ipsil. Choice

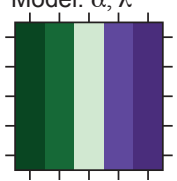

Model: $\alpha, \beta, \gamma, \lambda$

$\alpha$ Value Diff. (VD)

$\beta$ Timing ( $\mathrm{T}$ )

$\gamma$ VD, T Interaction

$\lambda$ Constant

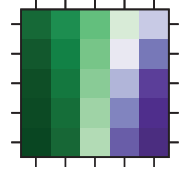

d

IS

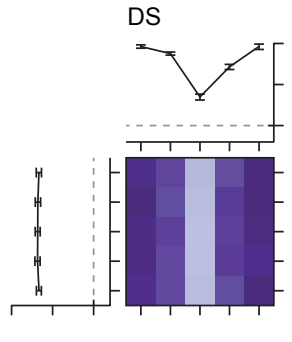

DS

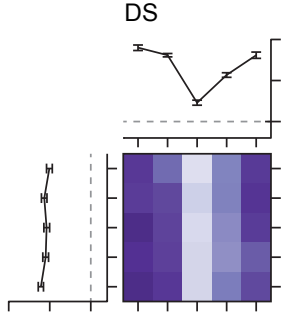

Model: $\alpha, \beta, \gamma, \lambda$

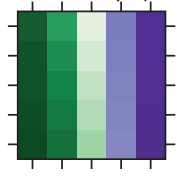




\section{Figure S3 (Pesaran)}

a

each choices

Monkey J

\section{Spiking Activity}
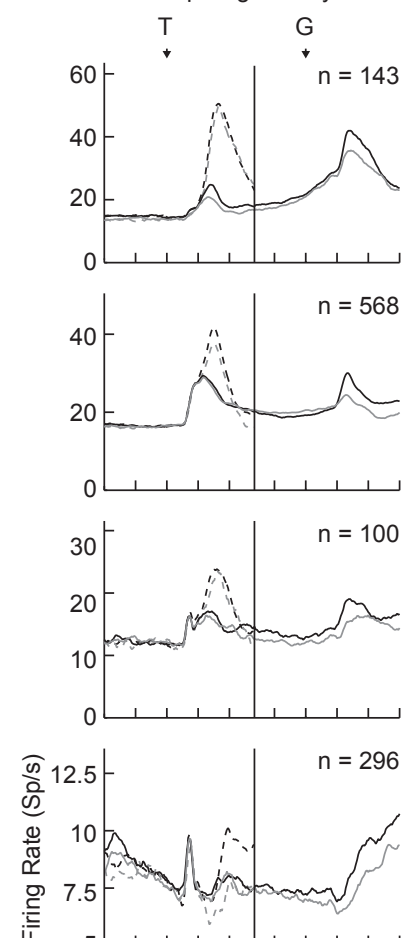

जी

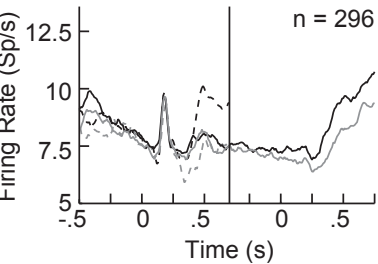

— Ipsil. Choice

— Delayed

_ Contral. Choice _. - Immediate
Condition Information
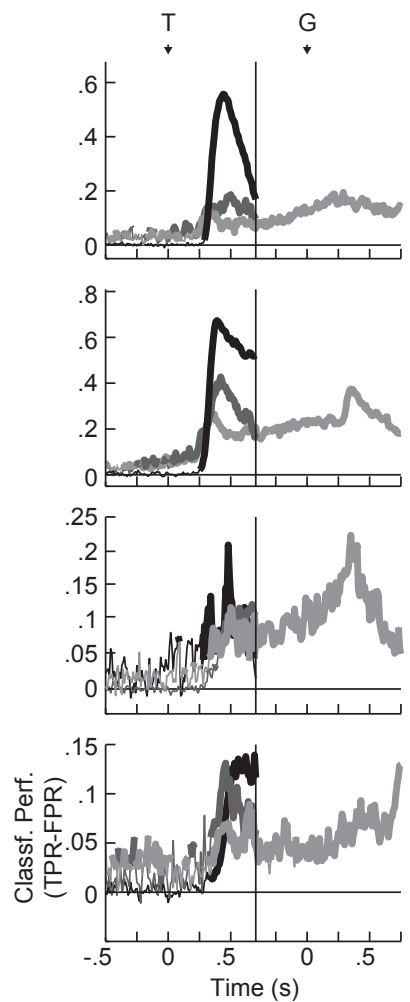

Classification

- IR vs DR

IR: Ipsi vs Contra
DR: Ipsi vs Contra

b

Reach choices Monkey H

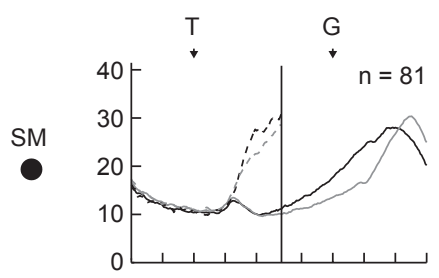

PMd

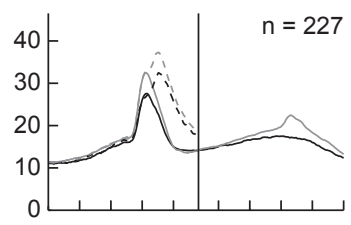

PMv

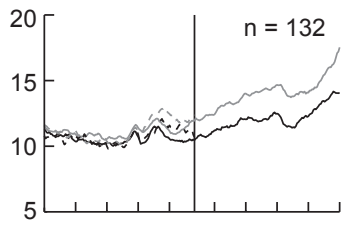

PFC
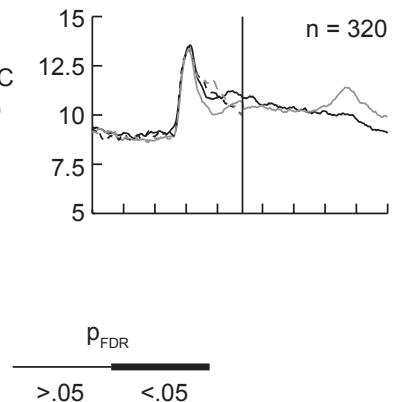
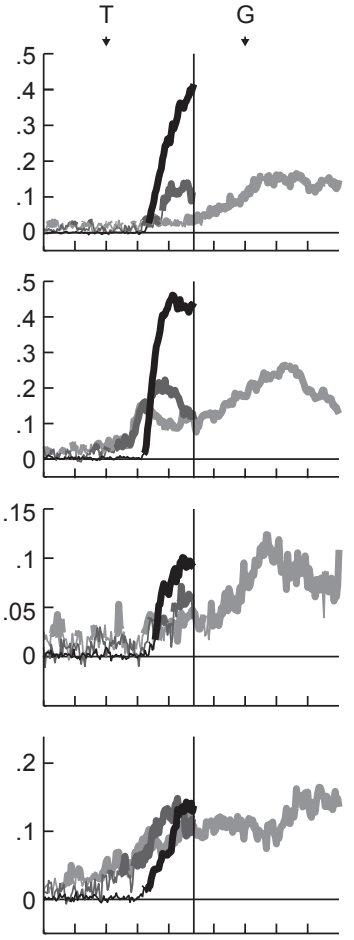


\section{Figure S4 (Pesaran)}

a

Saccade choices

Monkey J
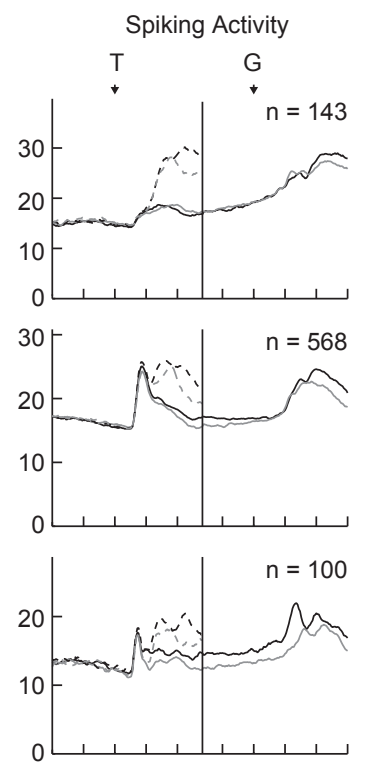

on

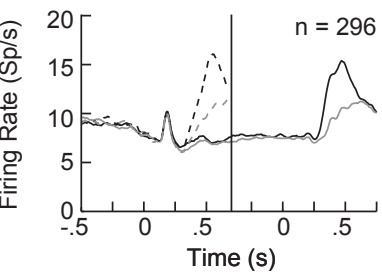

$\begin{array}{ll}\text { _ Ipsil. Choice } & \text { _ Delayed } \\ \text { — Contral. Choice } & \text {-- - Immediate }\end{array}$
Condition Information
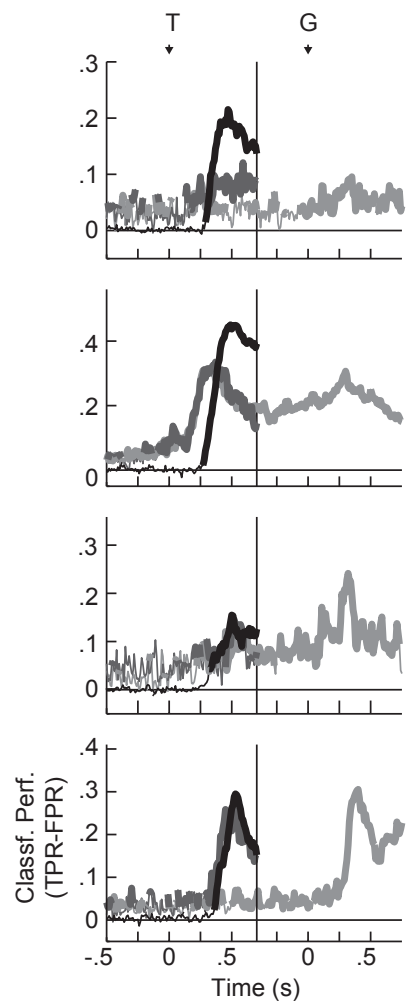

Classification

- IR vs DR

IR: Ipsi vs Contra

DR: Ipsi vs Contra b

Saccade choices Monkey H
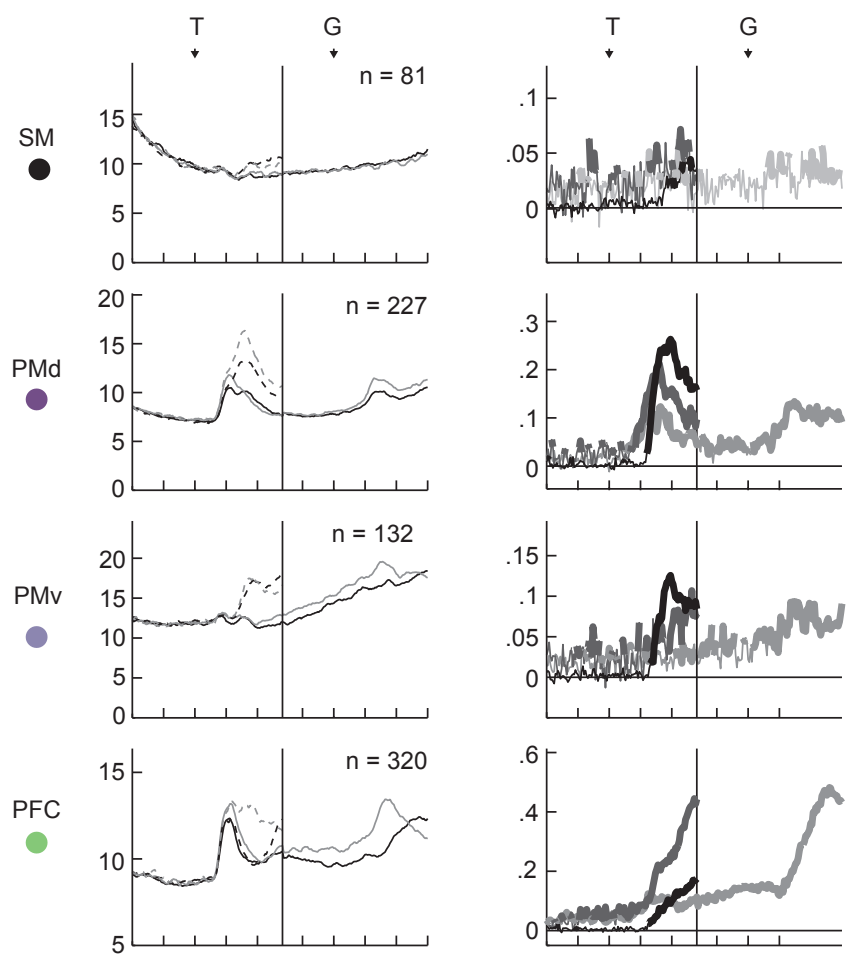
Figure S6 (Pesaran)

a

\section{Monkey J}
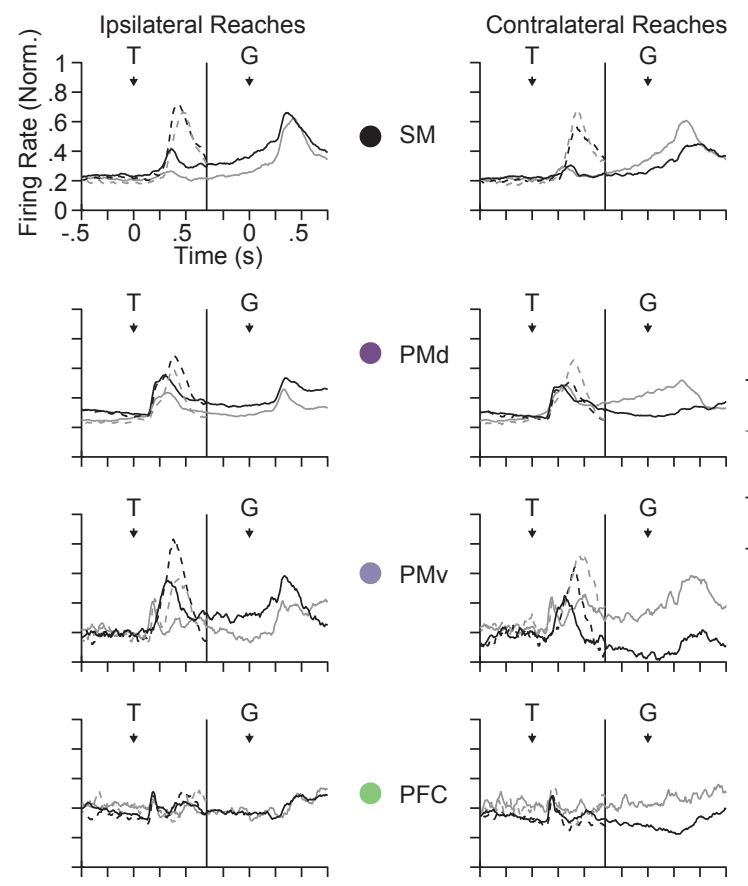

- Enc. Ipsi

- Enc. Contra

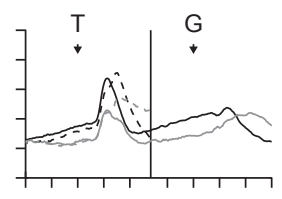

b

Monkey H

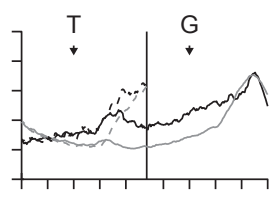

- Delayed

-. - Immediate
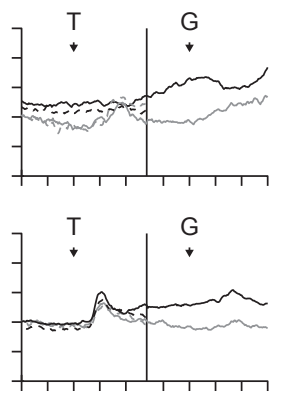
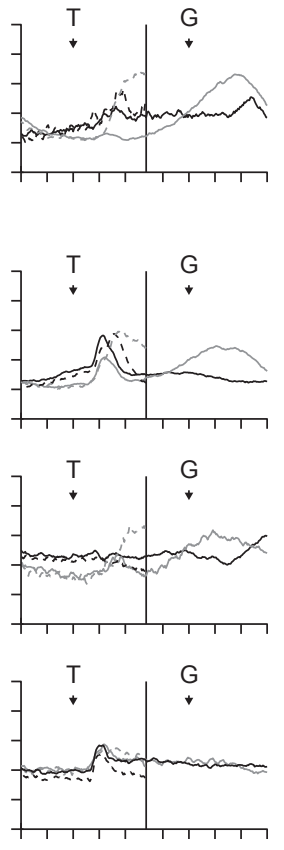
Figure S7 (Pesaran)

Monkey J
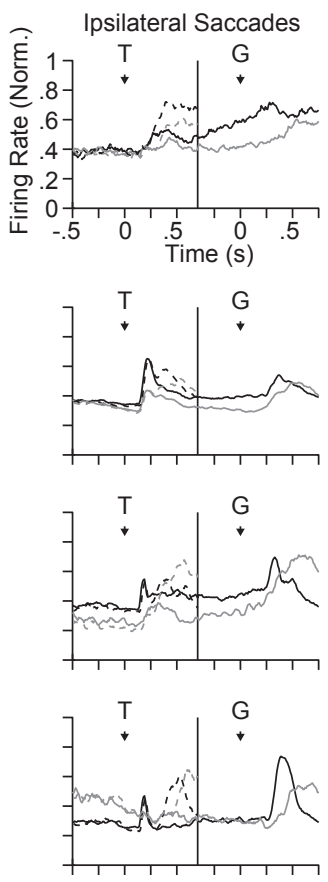

- $\mathrm{SM}$

Contralateral Saccades
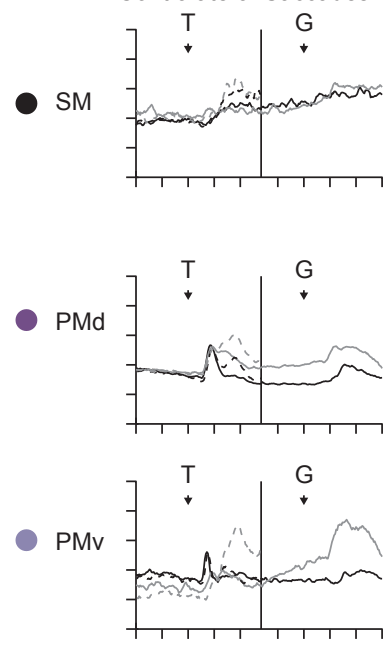

PFC

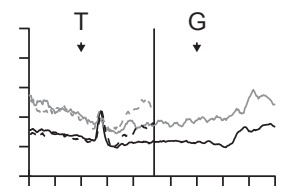

b

Monkey H
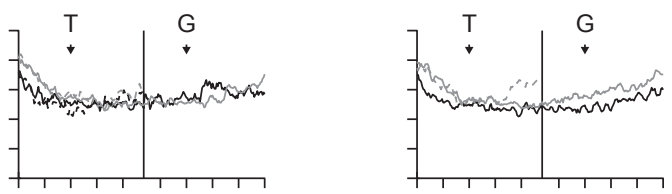

- Enc. Ipsi

- Enc. Contra

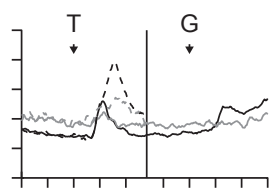

— Delayed

... Immediate
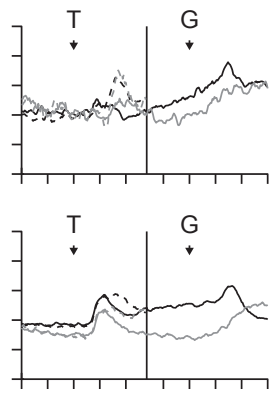
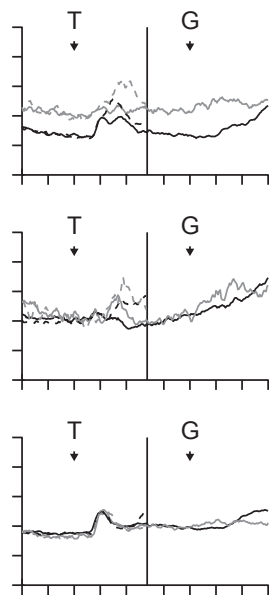


\section{Figure S8 (Pesaran)}

a

Monkey J, raw cross-classification

Reaches

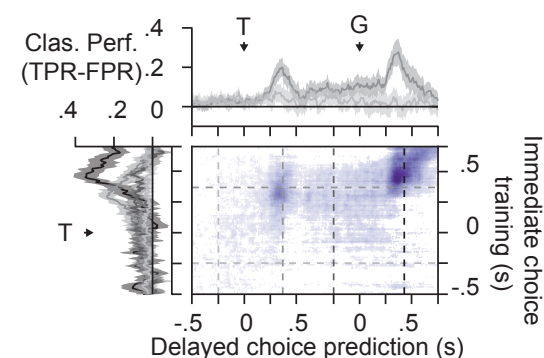

Delayed choice prediction (s)

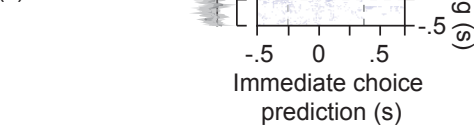

Saccades

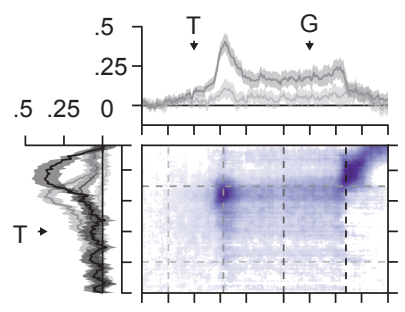

b Monkey $\mathrm{H}$, raw cross-classification

\section{Reaches}

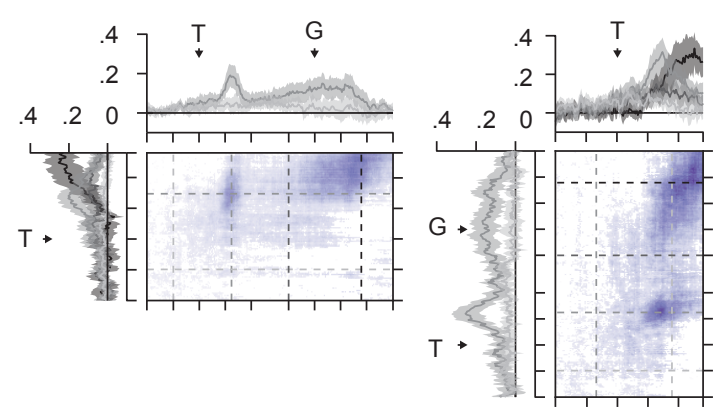

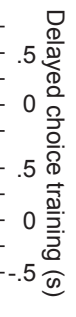

离

웅

.5 ळे

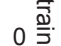

$5 \frac{0}{0}$

mediate choice

prediction (s)

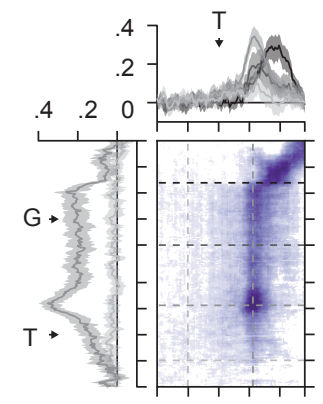

Classf. Perf. (TPR-FPR)

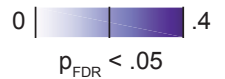

Saccades
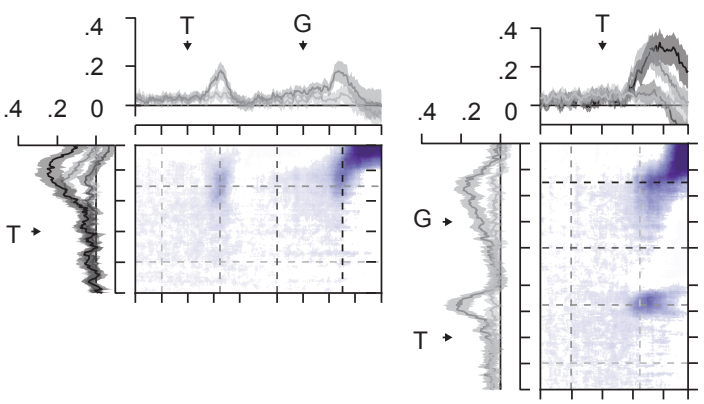

Timepoints of Interest

Baseline Commit

- Pre-Go

- Reaction Time 


\section{Figure S9 (Pesaran)}

a Similarity of choice representations Reaches

Monkey J

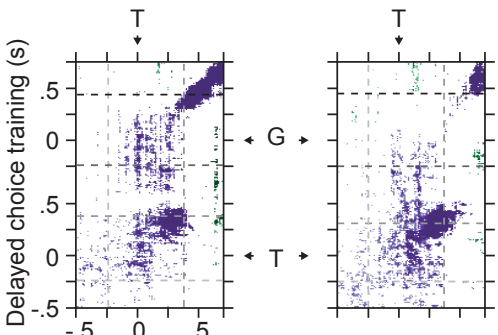

Immediate choice

prediction (s)

b

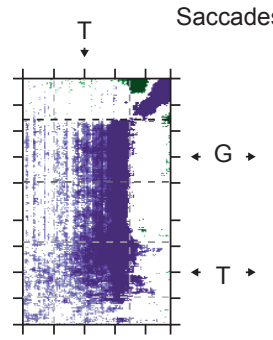

Monkey $\mathrm{H}$

\author{
- Baseline \\ Commit \\ - Pre-Go \\ RT
}

Dissimilar * Similar

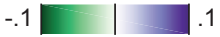

Corr. Classf. Perf.

$\mathrm{p}_{\mathrm{FDR}}<.05$ 